Homology, Homotopy and Applications, vol.18(1), 2016, pp.343-371

\title{
COMPLEX $N$-SPIN BORDISM OF SEMIFREE CIRCLE ACTIONS AND COMPLEX ELLIPTIC GENERA
}

\author{
M. NAEEM AHMAD \\ (communicated by Donald Davis)
}

\begin{abstract}
We give a complete bordism analysis of rational bordism groups of semifree circle actions on complex $N$-Spin manifolds. Moreover, we introduce the notion of a complex $N$-Spin ${ }^{c, t}$ manifold and give a characterization of cobordism groups of such manifolds which we use to compute the rational bordism groups of free circle actions of type $t$ on complex $N$-Spin manifolds. Furthermore, we exploit this bordism analysis to furnish a mechanism with which we investigate a description, in terms of kernels of complex elliptic genera, of the ideal $I_{*}^{N, t}$, generated by bordism classes of connected complex $N$-Spin manifolds admitting an effective circle action of type $t$, in the rational complex $N$ Spin cobordism ring $\Omega_{*}^{U, N} \otimes \mathbb{Q}$.
\end{abstract}

\section{Introduction}

Ochanine introduced elliptic genera in [16]. Landweber defined the classical elliptic genus of level 2 in [13]. This genus is intimately related with string theory (see, for instance, Witten $[\mathbf{2 0}, \mathbf{2 1}]$ ). The elliptic genus possesses a striking property which is its rigidity with respect to group actions. This was conjectured by Ochanine in [16], and by Witten in $[\mathbf{2 0}]$, where string theory arguments were used to support it. The rigidity of the elliptic genus was proved by Taubes in [18], Bott and Taubes in [2], and Liu in [14].

This work involves a study of bordism groups of semifree circle actions on complex $N$-Spin manifolds. The motivation for this study comes from a question of Witten [19]. He was interested in the Dirac operator with coefficients in the tangent bundle of a Spin manifold. He asked if the character valued index, for a circle action on a Spin manifold, is a constant. He further suggested that the problem can be investigated via the bordism groups of circle actions on Spin manifolds. The problem becomes simpler if attention is restricted to circle actions which are semifree. In [16], Ochanine determined the ideal in the rational Spin cobordism ring generated

Received March 10, 2014, revised July 13, 2015; published on May 18, 2016.

2010 Mathematics Subject Classification: Primary 57R85, 53C27, 57R77, 55N22, 58J26; Secondary 32Q60, 57R20, 55N20, 55P62, 55R10.

Key words and phrases: $U$-manifold, complex $N$-Spin manifold, complex elliptic genus of level $N$, the universal complex elliptic genus, circle action of type $t$, complex $N$-Spin ${ }^{c, t}$ manifold, complex twisted projective bundle.

Article available at http://dx.doi.org/10.4310/HHA.2016.v18.n1.a19

Copyright (C) 2016, International Press. Permission to copy for private use granted. 
by bordism classes of Spin manifolds admitting a semifree circle action of odd type by finding all multiplicative genera which vanish on it.

A complex $N$-Spin manifold is a manifold equipped with a stable complex structure on its tangent bundle and an $N$-th root of the associated complex line bundle. Hirzebruch defined bordism invariants $\varphi_{N}$ for these manifolds, which take values in modular forms of level $N$. He also showed in his rigidity theorem that these invariants vanish if such a manifold comes equipped with an $S^{1}$-action for which an invariant $t \in \mathbb{Z} / N$ called the "type" is non-zero (see [8]). Here the action is not required to be compatible with the $N$-Spin structure; in fact, $t$ can be thought of as the obstruction to a lift of the induced $S^{1}$-action on the principal $U(1)$-bundle (determined by the stable complex structure) to its $N$-fold covering (determined by the $N$-Spin structure).

Denoting by $I_{*}^{N, t}$ the ideal in the rational cobordism ring of complex $N$-Spin manifolds generated by connected complex $N$-Spin manifolds equipped with an effective $S^{1}$-action of type $t$, the above can be reformulated as $I_{*}^{N, t} \subset \operatorname{ker} \varphi_{N}$ for $t \not \equiv 0$ $(\bmod N)$. Noting that a complex $N$-Spin structure determines a complex $n$-Spin structure for $n \mid N$, it follows that $I_{*}^{N, t} \subset \bigcap_{n \mid N, n \nmid t} \operatorname{ker} \varphi_{n}$. In [9], Höhn showed that the equality holds when $t=1$ and based on that he conjectured that for all pairs $(N, t)$ with $N \geqslant 2$ and $t \not \equiv 0(\bmod N)$, we have

$$
I_{*}^{N, t}=\bigcap_{n \mid N, n \nmid t} \operatorname{ker} \varphi_{n} .
$$

In this work, we extend the investigation of this conjectural equation to higher values of $t$. In Section 2, we find a condition under which the conjecture holds true. That condition involves constructing bordism classes of complex $\mathrm{N}$-Spin manifolds satisfying a certain inclusion (see Proposition 2.4). In Section 3, we give a complete bordism analysis of rational bordism groups of semifree circle actions on complex $N$-Spin manifolds using classical geometric techniques. In Section 4, we invoke that analysis on the exactness of the sequence (3.1) to construct bordism classes satisfying the inclusion of Proposition 2.4 and verify that the conjectural equation (1.1) holds true for all values of $t$ with $N \leqslant 9$, except for the case $(N, t)=(6,3)$ which remains unanswered. Moreover, the technique developed in this work provides a way with which to investigate the conjectural equation for any values of $N$ and $t$.

This work is part of the author's Ph.D. thesis [1], written at Kansas State University in 2011. I would like to thank my thesis advisor, Professor Gerald Hoehn, for his ideas and several useful discussions.

\section{The universal complex elliptic genus}

Krichever showed in $[\mathbf{1 1}]$ that if the first Chern class of a unitary manifold is divisible by a whole number $k$, then the $A_{k}$-genus is rigid. (The generating series for the genus $A_{k}$, where $k=2,3,4, \ldots$, is of the form $k x e^{x} /\left(e^{k x}-1\right)$.) He proved in [12] that the universal complex elliptic genus is rigid for $S^{1}$-actions on $S U$-manifolds, that is, almost complex manifolds whose first Chern class is zero. Höhn studied the universal complex elliptic genus $\varphi_{\text {ell }}$ and rediscovered this result in [9]. The complex elliptic genus $\varphi_{N}$ of level $N$ factors over $\varphi_{\text {ell }}$. We will exploit this relationship to study the ideal $I_{*}^{N, t}$ in terms of kernels of elliptic genera. We begin by summarizing a few definitions and results from $[\mathbf{9}]$ needed to present the definition of $\varphi_{\text {ell }}$ and set 
up notation for the subsequent development of this article. Let $M$ be a connected $U$ manifold with the principal $S^{1}$-bundle $K$ associated to its principal stable tangential $U(n)$-bundle via the determinant representation $\Lambda^{n}: U(n) \rightarrow U(1) \cong S^{1}$. A complex $N$-Spin structure (or simply an $N$-structure) of $M$ is a pair $(E, \pi)$ consisting of a principal $S^{1}$-bundle $E$ over $M$ and a covering map $\pi: E \rightarrow K$ (of degree $N$ ) such that the diagram

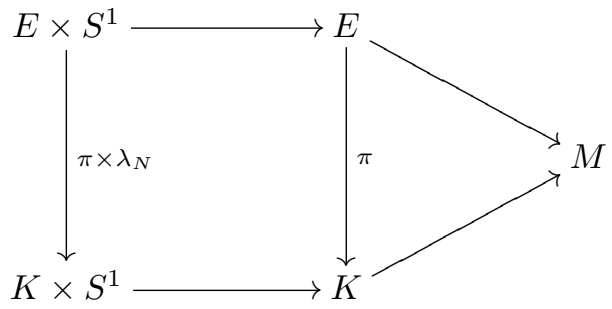

commutes, where $\lambda_{N}: S^{1} \rightarrow S^{1}$ is the canonical $N$-fold covering map given by $g \mapsto$ $g^{N}$, and the horizontal maps are the natural actions of $S^{1}$ on $E$ and $K$. Two complex $N$-Spin structures $(E, \pi)$ and $\left(E^{\prime}, \pi^{\prime}\right)$ of $M$ are said to be equivalent if there exists a principal $S^{1}$-bundle isomorphism $f: E^{\prime} \rightarrow E$ such that $\pi \circ f=\pi^{\prime}$. A connected $U$ manifold with an equivalence class of a complex $N$-Spin structure is called a complex $N$-Spin manifold (or simply an $N$-manifold). A $U$-manifold admits a complex $N$ Spin structure if and only if its first Chern class is divisible by $N$. The map $\Omega_{*}^{U, N} \rightarrow$ $\Omega_{*}^{U}$ from the complex $N$-Spin cobordism ring $\Omega_{*}^{U, N}$ to the $U$-cobordism $\operatorname{ring} \Omega_{*}^{U}$, defined by forgetting the complex $N$-Spin structure, becomes a ring isomorphism after tensoring with the rationals $\mathbb{Q}$. Now, let $\alpha: S^{1} \times M \rightarrow M$ be a smooth action of circle $S^{1}$ on the connected complex $N$-Spin manifold $M$ and for $\lambda \in S^{1}$ denote the differential of $\alpha(\lambda)$ on the tangent bundle $\tau(M)$ of $M$ by $\mathrm{D} \alpha(\lambda)$; then the type of the circle action $\alpha$ on the complex $N$-Spin manifold $M$ is the unique element $t \in \mathbb{Z} / N \mathbb{Z} \subset$ $S^{1}$ defined as follows: Let $p \in K$, and let $\gamma: S^{1} \rightarrow K$ given by $\lambda \mapsto \operatorname{det}(\operatorname{D} \alpha(\lambda) \oplus \operatorname{id}) p$ be a closed path. Let $q$ be an element of $E$ with $\pi(q)=p$. The type of the circle action $\alpha$ is the element $t \in \mathbb{Z} / N \mathbb{Z}$ such that $t \gamma^{\prime}(0)=\gamma^{\prime}(1)$, where $\gamma^{\prime}:[0,1] \rightarrow E$ is the unique lifting of $\gamma$ with $\pi \circ \gamma^{\prime}=\gamma$ and $\gamma^{\prime}(0)=q$. The type $t$ of a smooth circle action on a connected complex $N$-Spin manifold is independent of the chosen $q$, but it generally depends on the chosen complex $N$-Spin structure. However, if $[M] \neq 0$ in $\Omega_{*}^{U, N} \otimes \mathbb{Q}$, i.e., not all Chern numbers are zero, then the type of the circle action is independent of a chosen complex $N$-Spin structure and it is equal to the residue class modulo $N$ of the sum of rotation numbers of any fixed point component. For the ideal $I_{*}^{N, t}$ in $\Omega_{*}^{U, N} \otimes \mathbb{Q}$ generated by the connected complex $N$-Spin manifolds with an effective circle action of type $t$, we have $I_{*}^{N, t}=I_{*}^{N, \operatorname{gcd}(N, t)}$ for any positive integer $N$. Hence we will normally consider only those ideals $I_{*}^{N, t}$ for which an integer representing the class of $t$ is a divisor of $N$. Let $t$ and $s$ be two positive integers. By an abuse of notation we will also denote by $t$ and $s$ the equivalence classes of the integers $t$ and $s$ in $\mathbb{Z} / N \mathbb{Z}$, respectively. If $t \mid s$, then $I_{*}^{N, 1} \subset I_{*}^{N, t} \subset I_{*}^{N, s} \subset I_{*}^{N, 0}$.

For $n \mid N$, a connected complex $N$-Spin manifold $M$ with a circle action of type $t \not \equiv 0(\bmod N)$ is also an $n$-manifold with a circle action of the type $t \not \equiv 0(\bmod n)$ if $n \nmid t$. So, it follows by the rigidity theorem of Hirzebruch for elliptic genera of level $n$ (see $\left[8\right.$, p. 58]) that $[M] \in \bigcap_{n \mid N, n \nmid t} \operatorname{ker} \varphi_{n}$, where $\varphi_{n}$ denotes the complex elliptic 
genus of level $n$. Hence the ideals $I_{*}^{N, t}$ with $t \not \equiv 0(\bmod N)$ satisfy the inclusion $I_{*}^{N, t} \subset$ $\bigcap_{n \mid N, n \nmid t} \operatorname{ker} \varphi_{n}$. This inclusion will be used later in this section.

Let $\Lambda$ be a graded commutative algebra with identity over the rationals $\mathbb{Q}$. A complex genus $\varphi$ is a graded algebra homomorphism $\Omega_{*}^{U} \otimes \mathbb{Q} \rightarrow \Lambda$ so that $\varphi(1)=1$ holds. The Chern numbers of a complex $N$-Spin manifold specify its rational bordism class in $\Omega_{*}^{U, N} \otimes \mathbb{Q}$ and in $\Omega_{*}^{U} \otimes \mathbb{Q}$, and as noted above $\Omega_{*}^{U, N} \otimes \mathbb{Q} \cong \Omega_{*}^{U} \otimes \mathbb{Q}$, so one can identify the genera of $\Omega_{*}^{U, N} \otimes \mathbb{Q}$ and $\Omega_{*}^{U} \otimes \mathbb{Q}$ with one another. Analogous to the genera of $\Omega_{*}^{U} \otimes \mathbb{Q}$ with values in an algebra over $\mathbb{Q}$, one can also consider the genera of $\Omega_{*}^{U} \otimes \mathbb{C}$ with values in an algebra over $\mathbb{C}$. If $a_{1}, \ldots, a_{n} \in \mathbb{N}$, then the weighted projective space with weights $a_{1}, \ldots, a_{n} \in \mathbb{N}$ - denoted by $\mathbb{C P}^{a_{1}, \ldots, a_{n}}$ — is defined by $\left(\mathbb{C}^{n} \backslash\{0\}\right) / \sim$, where $\left(x_{1}, \ldots, x_{n}\right) \sim\left(y_{1}, \ldots, y_{n}\right)$ if and only if there exists a $\lambda \in \mathbb{C}^{*}$ with $y_{i}=\lambda^{a_{i}} x_{i}$ for all $i=1, \ldots, n$. Let $V$ (not necessarily irreducible) be a projective variety in $\mathbb{C P}^{a_{1}, \ldots, a_{n}}$ with weighted homogeneous annihilator ideal $I(V) \subset \mathbb{C}\left[x_{1}, \ldots, x_{n}\right]$, where $x_{i}$ has weight $a_{i}$, and $K(V)$ denotes the graded coordinate algebra $\mathbb{C}\left[x_{1}, \ldots, x_{n}\right] / I(V)$ of $V$ with the projection map $\pi: \mathbb{C}\left[x_{1}, \ldots, x_{n}\right] \rightarrow K(V)$. Now with these notations one can associate a genus to the variety $V$ as follows: Let $\left[X_{1}\right],\left[X_{2}\right], \ldots$ be a basis of $\Omega_{*}^{U} \otimes \mathbb{C}$, and let $a_{1}<a_{2}<\cdots<a_{n}$. The genus belonging to the variety $V$ is a graded algebra homomorphism $\varphi_{V}: \Omega_{*}^{U} \otimes \mathbb{C} \rightarrow K(V)$ defined by $\varphi_{V}=\pi \circ \lambda_{a_{1}, \ldots, a_{n}}$, where the map $\lambda_{a_{1}, \ldots, a_{n}}: \Omega_{*}^{U} \otimes \mathbb{C} \rightarrow \mathbb{C}\left[x_{1}, \ldots, x_{n}\right]$ is given by

$$
\left[X_{i}\right] \mapsto \begin{cases}x_{j} & \text { if } i=a_{j}, \\ 0 & \text { otherwise. }\end{cases}
$$

The genus $\varphi_{V}$ depends upon the choice of a basis sequence and the choice of a coordinate system for $\mathbb{C P}^{a_{1}, \ldots, a_{n}}$. Let $V$ and $W$ be two projective varieties in $\mathbb{C P}^{a, \ldots, a_{n}}$ with a fixed coordinate system. Then with a fixed basis sequence one can see that $\operatorname{ker} \varphi_{V \cup W}=\operatorname{ker} \varphi_{V} \cap \operatorname{ker} \varphi_{W}$.

The universal complex elliptic genus is the genus belonging to the power series which is the solution of a certain second order differential equation defined as follows: Let $S(y)=y^{4}+q_{1} y^{3}+q_{2} y^{2}+q_{3} y+q_{4}$ be a standard polynomial in $y$ with coefficients $q_{1}$ to $q_{4}$, and let $Q(x)=1+a_{1} x+a_{2} x^{2}+\cdots$ be a power series in $x$. Let $h(x):=$ $\frac{f^{\prime}(x)}{f(x)}$ be the logarithmic derivative of $f(x)=\frac{x}{Q(x)}$. It was shown in [9, Lemma 2.2.1] that the second order differential equation

$$
\left(h^{\prime}(x)\right)^{2}=S(h(x))
$$

has a unique solution $h(x) \in \mathbb{Q}\left[q_{1}, q_{2}, q_{3}, q_{4}\right][[x]]\left[x^{-1}\right]$, which in turn determines the power series $Q(x) \in \mathbb{Q}\left[q_{1}, q_{2}, q_{3}, q_{4}\right][[x]]$. Furthermore, if $q_{1}$ to $q_{4}$ are assigned weights 1 to 4 , then the coefficients $a_{n}$ of $Q(x)$ are homogeneous polynomials of weight $n$ in $q_{1}$ to $q_{4}$. The universal complex elliptic genus

$$
\varphi_{\text {ell }}: \Omega_{*}^{U} \otimes \mathbb{Q} \rightarrow \mathbb{Q}\left[q_{1}, q_{2}, q_{3}, q_{4}\right]
$$

is the complex elliptic genus having the characteristic power series $Q(x)$ belonging to the solution of the differential equation (2.1). A basis sequence $\left[W_{1}\right],\left[W_{2}\right],\left[W_{3}\right],\left[W_{4}\right]$, $\left[W_{5}\right], \ldots$ of $\Omega_{*}^{U} \otimes \mathbb{Q}$ was constructed in [9] on which the universal complex elliptic genus takes the values: $\varphi_{\text {ell }}\left(\left[W_{1}\right]\right)=A, \varphi_{\text {ell }}\left(\left[W_{2}\right]\right)=B, \varphi_{\text {ell }}\left(\left[W_{3}\right]\right)=C, \varphi_{\text {ell }}\left(\left[W_{4}\right]\right)=D$, and $\varphi_{\text {ell }}\left(\left[W_{n}\right]\right)=0$ for all $n \geqslant 5$, where the indeterminates $A=\frac{1}{2} q_{1}, B=\frac{3}{2} q_{1}^{2}-4 q_{2}$, $C=\frac{1}{32} q_{1}^{3}-\frac{1}{8} q_{1} q_{2}+\frac{1}{4} q_{3}$, and $D=\frac{3}{128} q_{1}^{4}-\frac{1}{8} q_{1}^{2} q_{2}+\frac{1}{8} q_{1} q_{3}+\frac{1}{8} q_{2}^{2}-\frac{1}{2} q_{4}$ are provided 
with the weights 1 to 4 . Conversely, if $A$ to $D$ are given, then one can determine $q_{1}$ to $q_{4}$ as: $q_{1}=2 A, q_{2}=\frac{3}{2} A^{2}-\frac{1}{4} B, q_{3}=\frac{1}{2} A^{3}-\frac{1}{4} A B+4 C$, and $q_{4}=\frac{1}{16} A^{4}-\frac{1}{16} A^{2} B+$ $2 A C+\frac{1}{64} B^{2}-2 D$. With respect to this homogeneous change of coordinates we will consider the universal complex elliptic genus

$$
\varphi_{\text {ell }}: \Omega_{*}^{U} \otimes \mathbb{Q} \rightarrow \mathbb{Q}[A, B, C, D]
$$

as the complex genus with values in the graded rational algebra $\mathbb{Q}[A, B, C, D]$. Let $\mathbb{C}[A, B, C, D]$ be the graded coordinate algebra of the twisted projective space $\mathbb{C P}^{1,2,3,4}$. The universal complex elliptic genus can also be regarded as the genus

$$
\varphi_{\text {ell }}: \Omega_{*}^{U} \otimes \mathbb{C} \rightarrow \mathbb{C}[A, B, C, D]
$$

belonging to the weighted projective variety $\mathbb{C P}^{1,2,3,4}$ and a basis $\left[W_{1}\right],\left[W_{2}\right],\left[W_{3}\right]$, $\left[W_{4}\right],\left[W_{5}\right],\left[V_{6}\right], \ldots$, where $\left[V_{n}\right]$ from $\operatorname{ker} \varphi_{\text {ell }} \mid \Omega_{n}^{U} \otimes \mathbb{C}$ are the bordism classes of manifolds such that the Milnor numbers $s_{n}\left(\left[V_{n}\right]\right) \neq 0$ for all $n \geqslant 6$.

Let $\mathbb{H}=\{z \in \mathbb{C}: \operatorname{Im}(z)>0\}$ be the complex upper half plane, and let $\tau \in \mathbb{H}$. Consider the lattice $L=2 \pi i(\mathbb{Z} \tau+\mathbb{Z})$ in $\mathbb{C}$, and let $\alpha \in \mathbb{C} / L$ be a non-zero $N$-division point of the associated elliptic curve. There is a unique elliptic function $h(x)$ of $L$ with the divisor $N \cdot(0)-N \cdot(\alpha)$ and the normalization $h(x)=x^{N}+O\left(x^{N+1}\right)$ of the Taylor expansion around the origin. The function $f(x)=\sqrt[N]{h(x)}$ is well-defined if $f(x)=x+O\left(x^{2}\right)$. The complex elliptic genus of level $N$ - denoted by $\varphi_{N}$ is the complex elliptic genus having the characteristic power series $Q(x)=\frac{x}{f(x)}=$ $1+b_{1} x+b_{2} x^{2}+\cdots$, where $f(x)$ as above is the function belonging to the lattice $L=2 \pi i(\mathbb{Z} \tau+\mathbb{Z})$ and the primitive $N$-division point $\frac{2 \pi i}{N}$. The modular subgroup

$$
\Gamma_{1}(N):=\left\{\left(\begin{array}{ll}
a & b \\
c & d
\end{array}\right) \in S L_{2}(\mathbb{Z}):\left(\begin{array}{ll}
a & b \\
c & d
\end{array}\right) \equiv\left(\begin{array}{ll}
1 & b \\
0 & 1
\end{array}\right) \quad(\bmod N)\right\}
$$

of $S L_{2}(\mathbb{Z})$ acts on $\mathbb{H}$ under the action $\sigma: S L_{2}(\mathbb{Z}) \times \mathbb{H} \rightarrow \mathbb{H}$ given by the map which sends $\left(\left(\begin{array}{ll}a & b \\ c & d\end{array}\right), \tau\right)$ to $\frac{a \tau+b}{c \tau+d}$. The orbit space of this action is denoted by $\mathbb{H} / \Gamma_{1}(N)$. The function $f(x)$ in the definition of $\varphi_{N}$ is determined by either of the following two differential equations: The first differential equation is of order 2 and has the form

$$
\left(\frac{f^{\prime}}{f}\right)^{\prime 2}=S\left(\frac{f^{\prime}}{f}\right)
$$

where $S(y)=y^{4}+q_{1} y^{3}+q_{2} y^{2}+q_{3} y+q_{4}$ is a polynomial of degree 4 , and the coefficients $q_{i}$ are modular forms of weight $i$ for $\Gamma_{1}(N)$. Since the power series $f(x)$ corresponding to $\varphi_{N}$ satisfies the differential equation (2.1) with special $q_{i}$, the complex elliptic genus $\varphi_{N}$ of level $N$ factorizes over $\varphi_{\text {ell }}$. The second differential equation is of order 2 and has the form

$$
\frac{1}{f^{N}}+d_{2 N} f^{N}=T_{N}\left(\frac{f^{\prime}}{f}\right)
$$

where $T_{N}(y)=y^{N}+d_{1} y^{N-1}+\cdots+d_{N-1} y+d_{N}$ is a polynomial of degree $N$ and $d_{i}$ are modular forms of weights $i$ for $\Gamma_{1}(N)$. The polynomial $T_{N}(y)$ is a so-called Zolotarev-polynomial. It is characterized by the equations $d_{N-1}=0$, and $T_{N}^{2}(y)=$ $4 d_{2 N}$ for $y \neq 0$ with $T_{N}^{\prime}(y)=0$, and another equation which gives a relationship 
between $S(y)$ and $T_{N}(y)$ given by $S(y) T_{N}^{\prime}(y)^{2}=N^{2} y^{2}\left(T_{N}(y)^{2}-4 d_{2 N}\right)$. This differential equation gives rise to two (depending on $N$ ) relations $R_{N-1}$ and $R_{N+1}$ which are weighted homogeneous in the coefficients $q_{1}$ to $q_{4}$ of $S(y)$. Note that the relations $R_{N-1}$ and $R_{N+1}$ can also be considered weighted homogeneous in the indeterminates $A$ to $D$. We will denote the variety $V\left(\left\langle R_{N-1}, R_{N+1}\right\rangle\right) \subset \mathbb{C P}^{1,2,3,4}$ by $C_{N}$. The mapping

$$
\Phi: \bigcup_{n \mid N, n>1} \overline{\mathbb{H} / \Gamma_{1}(n)} \rightarrow \mathbb{C P}^{1,2,3,4}
$$

which for each $n \mid N, n>1$ assigns to a pair $(\tau, n)$ the point $\left(q_{1}: q_{2}: q_{3}: q_{4}\right)$, where $q_{1}$ to $q_{4}$ are the coefficients of the polynomial $S(y)$ of the differential equation (2.2), is surjective to the curve $C_{N}$. The images $\Phi\left(\overline{\mathbb{H} / \Gamma_{1}(n)}\right)$ form a decomposition of $C_{N}$ into irreducible components. The image $\Phi\left(\overline{\mathbb{H} / \Gamma_{1}(n)}\right)$ is independent of $N$ (see $[\mathbf{1 0}$, Chapter 3]). We will denote the projection $\mathbb{C}[A, B, C, D] \longrightarrow \mathbb{C}[A, B, C, D] / I\left(C_{N}\right)$ by $\pi_{N}$. The kernel of $\pi_{N}$ is the annihilator ideal $I\left(C_{N}\right)$ of the curve $C_{N}$, simply denoted by $I_{N}$, and therefore by the Hilbert Nullstellensatz the radical of $\left\langle R_{N-1}, R_{N+1}\right\rangle$. It was shown in [9, Theorem 2.3.5] that $\left\langle R_{N-1}, R_{N+1}\right\rangle$ is a radical ideal. It has the primary ideal decomposition

$$
I_{N}=\bigcap_{n \mid N, n>1} P_{n},
$$

where the ideal $P_{n}$ corresponds to the irreducible component $\Phi\left(\overline{\mathbb{H} / \Gamma_{1}(n)}\right)$ of the curve $C_{N}$. The ideal $P_{n}$ is independent of the multiples $N$ of $n$. The complex elliptic genus $\varphi_{N}$ of level $N$ becomes the composition

$$
\Omega_{*}^{U} \otimes \mathbb{Q} \stackrel{\varphi_{\text {ell }}}{\longrightarrow} \mathbb{Q}[A, B, C, D] \stackrel{\pi_{N}}{\longrightarrow} \mathbb{Q}[A, B, C, D] / P_{N},
$$

where $\pi_{N}$ denotes the projection map.

Definition 2.1. For $t \mid N$, we define the ideal $P^{N, t}$ as the following intersection of ideals

$$
P^{N, t}=\bigcap_{n \mid N, n \nmid t} P_{n}
$$

For the first few ideals $P^{N, t}$ we have ${ }^{1}$

$$
\begin{aligned}
P^{2,1}= & \langle C, A\rangle, \\
P^{3,1}= & \left\langle 125 B^{2}+1152 A C-384 D, 18 A^{2}-B\right\rangle, \\
P^{4,1}= & \left\langle 125 B^{2} C+768 A C^{2}-384 C D, 125 A B^{2}+768 A^{2} C-384 A D,\right. \\
& \left.8 A^{3}-A B+4 C\right\rangle, \\
P^{4,2}= & \left\langle 2 A C-D, 8 A^{3}-A B+4 C\right\rangle, \\
P^{5,1}= & \left\langle 15625 B^{4}+480000 A B^{2} C+1843200 A^{2} C^{2}+36864 B C^{2}-96000 B^{2} D\right. \\
& -1474560 A C D+147456 D^{2}, 6250 A^{2} B^{2}-125 B^{3}+32000 A^{3} C \\
& -640 A B C+4096 C^{2}-19200 A^{2} D+384 B D, 1250 A^{4}-250 A^{2} B+67 B^{2} \\
& +1600 A C-192 D\rangle .
\end{aligned}
$$

${ }^{1}$ The computations were accomplished with the help of computer programs in Mathematica and MAGMA. 
Definition 2.2. Let $\left[W_{1}\right],\left[W_{2}\right],\left[W_{3}\right],\left[W_{4}\right],\left[V_{5}\right],\left[V_{6}\right], \ldots$ be a basis sequence of $\Omega_{*}^{U} \otimes$ $\mathbb{C}$ with $\left\langle\left[V_{5}\right],\left[V_{6}\right] \ldots\right\rangle=\operatorname{ker} \varphi_{\text {ell }}$ and $\left[W_{1}\right]$ to $\left[W_{4}\right]$ having the same meaning as above in this section. We define by $\psi_{N, t}$ the genus belonging to the variety $C_{N, t}=V\left(P^{N, t}\right) \subset$ $\mathbb{C P}^{1,2,3,4}$.

The definition of $\psi_{N, t}$ is independent of the chosen basis manifolds from $\operatorname{ker} \varphi_{\text {ell }}$, and the following situation exists in the $A, B, C, D$-coordinate system for $\mathbb{C P}^{1,2,3,4}$ :

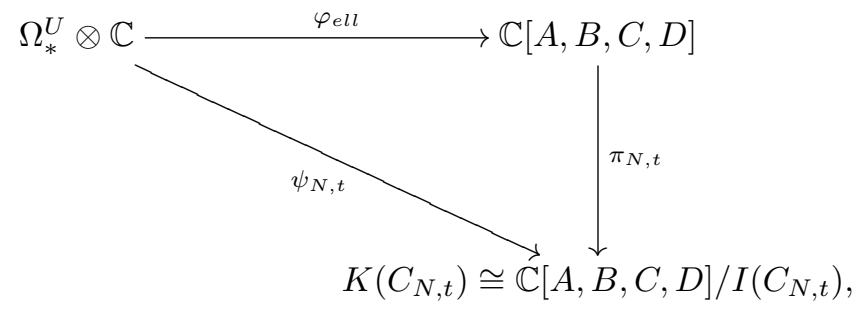

that is,

$$
\psi_{N, t}=\pi_{N, t} \circ \varphi_{e l l} .
$$

Proposition 2.3. We have $\operatorname{ker} \psi_{N, t}=\bigcap_{n \mid N, n \nmid t} \operatorname{ker} \varphi_{n}$.

Proof. Observe that

$$
\operatorname{ker} \psi_{N, t}=\operatorname{ker} \varphi_{C_{N, t}}=\bigcap_{n \mid N, n \nmid t} \operatorname{ker} \varphi_{C_{n}}=\bigcap_{n \mid N, n \nmid t} \operatorname{ker} \varphi_{\Phi\left(\overline{\mathbb{H}} / \Gamma_{1}(n)\right)} .
$$

Now $[X] \in \operatorname{ker} \varphi_{\Phi\left(\overline{\mathbb{H} / \Gamma_{1}(n)}\right)} \Leftrightarrow \varphi_{\text {ell }}([X]) \in I\left(\Phi\left(\overline{\mathbb{H} / \Gamma_{1}(n)}\right)\right)$. The point $P \in \Phi\left(\overline{\mathbb{H} / \Gamma_{1}(n)}\right)$ corresponding to $\tau \in \overline{\mathbb{H} / \Gamma_{1}(n)}$ has the parametrization:

$$
P(\tau)=(A(\tau): B(\tau): C(\tau): D(\tau)) .
$$

Hence $\varphi_{\text {ell }}([X])(P(\tau))=\varphi_{n}([X])(\tau)$, if $\varphi_{\text {ell }}([X]) \in I\left(\Phi\left(\overline{\mathbb{H} / \Gamma_{1}(n)}\right)\right) \Leftrightarrow \varphi_{n}([X])=0$. Thus the result follows.

Now we find a condition under which the conjectural equation (1.1) is true:

Proposition 2.4. If there exist bordism classes $\left[V_{1}\right],\left[V_{2}\right], \ldots,\left[V_{m}\right] \in I_{*}^{N, t}$ such that

$$
P^{N, t} \subset\left\langle\varphi_{\text {ell }}\left[V_{1}\right], \varphi_{\text {ell }}\left[V_{2}\right], \ldots, \varphi_{\text {ell }}\left[V_{m}\right]\right\rangle
$$

then we have

$$
I_{*}^{N, t}=\bigcap_{n \mid N, n \nmid t} \operatorname{ker} \varphi_{n} .
$$

Proof. Let the sequence $\left[W_{5}\right],\left[W_{6}\right], \ldots$ have the same meaning as above in this section, then $\operatorname{ker} \varphi_{\text {ell }}=\left\langle\left[W_{5}\right],\left[W_{6}\right], \ldots\right\rangle$. It was shown by Höhn in $[\mathbf{9}]$ that $\left\langle\left[W_{5}\right],\left[W_{6}\right], \ldots\right\rangle \subset$ $I_{*}^{N, 1} \subset I_{*}^{N, t}$ for all $t \neq 0$. So we get $\left\langle\left[V_{1}\right],\left[V_{2}\right], \ldots\left[V_{m}\right],\left[W_{5}\right],\left[W_{6}\right], \ldots\right\rangle \subset I_{*}^{N, t}$. As noted above in this section, we have the inclusion

$$
I_{*}^{N, t} \subset \bigcap_{n \mid N, n \nmid t} \operatorname{ker} \varphi_{n} .
$$

Hence by Proposition 2.3, we get

$$
\left\langle\left[V_{1}\right],\left[V_{2}\right], \ldots\left[V_{m}\right],\left[W_{5}\right],\left[W_{6}\right], \ldots\right\rangle \subset I_{*}^{N, t} \subset \bigcap_{n \mid N, n \nmid t} \operatorname{ker} \varphi_{n}=\operatorname{ker}\left(\psi_{N, t}\right) .
$$

Now it remains to show that $\operatorname{ker}\left(\psi_{N, t}\right) \subset\left\langle\left[V_{1}\right],\left[V_{2}\right], \ldots\left[V_{m}\right],\left[W_{5}\right],\left[W_{6}\right], \ldots\right\rangle$. Let $[V]$ be a bordism class in $\operatorname{ker}\left(\psi_{N, t}\right)$. Since by equation $(2.4) \psi_{N, t}=\pi_{N, t} \circ \varphi_{\text {ell }}$, we have 
$\varphi_{\text {ell }}([V]) \in \operatorname{ker}\left(\pi_{N, t}\right)$, and hence $\varphi_{\text {ell }}([V]) \in P^{N, t}$, and so $\varphi_{\text {ell }}([V]) \in\left\langle\varphi_{\text {ell }}\left[V_{1}\right], \varphi_{\text {ell }}\left[V_{2}\right]\right.$, $\left.\ldots \varphi_{\text {ell }}\left[V_{m}\right]\right\rangle$, by hypothesis. Since $\varphi_{\text {ell }}$ is a homomorphism, there exists a $[U] \in$ $\left\langle\left[V_{1}\right],\left[V_{2}\right], \ldots\left[V_{m}\right]\right\rangle$ such that $\varphi_{\text {ell }}([V])=\varphi_{\text {ell }}([U])$ which implies $[V]-[U] \in \operatorname{ker}\left(\varphi_{\text {ell }}\right)$ $=\left\langle\left[W_{5}\right],\left[W_{6}\right], \ldots\right\rangle$. Hence $[V] \in[U]+\left\langle\left[W_{5}\right],\left[W_{6}\right], \ldots\right\rangle \subset\left\langle\left[V_{1}\right],\left[V_{2}\right], \ldots,\left[V_{m}\right],\left[W_{5}\right]\right.$, $\left.\left[W_{6}\right], \ldots\right\rangle$. This completes the proof.

In Section 4, we will verify the conjectural equation (1.1) for several cases of pairs $(N, t)$, by constructing bordism classes satisfying the condition of Proposition 2.4, after we introduce certain notions and prove our main results pertaining to bordism theory of complex $N$-Spin manifolds with circle actions in Section 3 required for our work with proofs of those cases of the conjectural equation.

\section{Bordism of semifree circle actions}

This section is devoted to the study of smooth circle actions on complex $N$-Spin manifolds from the bordism theory viewpoint. A smooth circle action on a smooth manifold is called free if all its isotropy groups are the unit subgroup of $S^{1}$ and semifree if they are either $S^{1}$ or the unit subgroup of $S^{1}$. In what follows, we will tacitly assume that a circle action on a $U$-manifold preserves its $U$-structure, and we will denote by:

(i) $\Omega_{n}^{U}(F)$ the bordism group of all closed $n$-dimensional $U$-manifolds with free circle actions,

(ii) $\Omega_{n}^{U}(S F)$ the bordism group of all closed $n$-dimensional $U$-manifolds with semifree circle actions, and

(iii) $\Omega_{n}^{U}(S F, F)$ the bordism group of all compact $n$-dimensional $U$-manifolds with semifree circle actions which are free on the boundary.

Also, we will use notations $\Omega_{n}^{U, N}(F), \Omega_{n}^{U, N}(S F)$, and $\Omega_{n}^{U, N}(S F, F)$ for similar bordism groups of complex $N$-Spin manifolds with circle actions which preserve their underlying $U$-structures, as well as notations $\Omega_{n}^{U, N, t}(F), \Omega_{n}^{U, N, t}(S F)$, and $\Omega_{n}^{U, N, t}(S F, F)$ for bordism groups of complex $N$-Spin manifolds with such circle actions having a fixed type $t$.

It was noted in $[7]$ that the sequence

$$
\cdots \longrightarrow \Omega_{n}^{U}(F) \stackrel{i_{U}}{\longrightarrow} \Omega_{n}^{U}(S F) \stackrel{j_{U}}{\longrightarrow} \Omega_{n}^{U}(S F, F) \stackrel{\partial_{U}}{\longrightarrow} \Omega_{n-1}^{U}(F) \longrightarrow \cdots
$$

is exact, where the maps $i_{U}, j_{U}$, and $\partial_{U}$ have the usual meanings (see, for instance, [7]).

Analogously, the sequence

$$
\cdots \longrightarrow \Omega_{n}^{U, N}(F) \stackrel{i_{N}}{\longrightarrow} \Omega_{n}^{U, N}(S F) \stackrel{j_{N}}{\longrightarrow} \Omega_{n}^{U, N}(S F, F) \stackrel{\partial_{N}}{\longrightarrow} \Omega_{n-1}^{U, N}(F) \longrightarrow \cdots
$$

is exact, where the maps $i_{N}, j_{N}$, and $\partial_{N}$ have the same meanings as in the above sequence.

Recall from Section 2 that the type of a circle action on a connected complex $N$-Spin manifold is an element $t$ of the group $\mathbb{Z} / N \mathbb{Z}$. We have the decompositions:

$$
\Omega_{n}^{U, N}(F)=\bigoplus_{t \in \mathbb{Z} / N \mathbb{Z}} \Omega_{n}^{U, N, t}(F), \quad \Omega_{n}^{U, N}(S F)=\bigoplus_{t \in \mathbb{Z} / N \mathbb{Z}} \Omega_{n}^{U, N, t}(S F), \quad \text { and }
$$




$$
\Omega_{n}^{U, N}(S F, F)=\bigoplus_{t \in \mathbb{Z} / N \mathbb{Z}} \Omega_{n}^{U, N, t}(S F, F),
$$

where the groups $\Omega_{n}^{U, N, t}(F), \Omega_{n}^{U, N, t}(S F)$, and $\Omega_{n}^{U, N, t}(S F, F)$ are defined by requiring circle actions to be of type $t$. Furthermore, each map in the above sequence preserves the type $t$ of circle actions, and so the sequence

$$
\cdots \longrightarrow \Omega_{n}^{U, N, t}(F) \stackrel{i_{N, t}}{\longrightarrow} \Omega_{n}^{U, N, t}(S F) \stackrel{j_{N, t}}{\longrightarrow} \Omega_{n}^{U, N, t}(S F, F) \stackrel{\partial_{N, t}}{\longrightarrow} \Omega_{n-1}^{U, N, t}(F) \longrightarrow \cdots
$$

is exact, where the maps $i_{N, t}, j_{N, t}$, and $\partial_{N, t}$ are the restrictions to the groups $\Omega_{n}^{U, N, t}(F), \Omega_{n}^{U, N, t}(S F)$, and $\Omega_{n}^{U, N, t}(S F, F)$ of the maps $i_{N}, j_{N}$, and $\partial_{N}$, respectively.

The above three sequences remain exact after tensoring with the rationals $\mathbb{Q}$. In particular, we will use the exactness of the sequence

$$
\begin{array}{cc}
\cdots \longrightarrow & \Omega_{n}^{U, N, t}(F) \otimes \mathbb{Q} \stackrel{i_{N, t}}{\longrightarrow} \Omega_{n}^{U, N, t}(S F) \otimes \mathbb{Q} \stackrel{j_{N, t}}{\longrightarrow} \Omega_{n}^{U, N, t}(S F, F) \otimes \mathbb{Q} \\
\stackrel{\partial_{N, t}}{\longrightarrow} & \Omega_{n-1}^{U, N, t}(F) \otimes \mathbb{Q} \longrightarrow \\
\longrightarrow & \cdots
\end{array}
$$

to construct bordism classes satisfying the condition of Proposition 2.4, where we denote by an abuse of notation the maps $i_{N, t} \otimes \mathbb{Q}, j_{N, t} \otimes \mathbb{Q}$, and $\partial_{N, t} \otimes \mathbb{Q}$ by $i_{N, t}, j_{N, t}$, and $\partial_{N, t}$, respectively. In the remainder of this section we will do bordism analysis to find computable characterizations of the groups $\Omega_{n}^{U, N, t}(S F, F) \otimes \mathbb{Q}$ and $\Omega_{n}^{U, N, t}(F) \otimes$ $\mathbb{Q}$ needed to make calculations to construct those bordism classes. We will begin by studying the groups $\Omega_{n}^{U}(S F, F)$ and $\Omega_{n}^{U, N}(S F, F)$, and then we will rationalize them to find a characterization of the group $\Omega_{n}^{U, N}(S F, F) \otimes \mathbb{Q}$ which will lead to a computable characterization of the group $\Omega_{n}^{U, N, t}(S F, F) \otimes \mathbb{Q}$. After that we will introduce and develop the notion of a complex $N$-Spin ${ }^{c, t}$ structure needed to find a computable characterization of the group $\Omega_{n}^{U, N, t}(F) \otimes \mathbb{Q}$.

\subsection{The group $\Omega_{n}^{U}(S F, F)$}

In this subsection we will study the group $\Omega_{n}^{U}(S F, F)$ of bordism classes of compact $n$-dimensional $U$-manifolds with semifree circle actions which are free on the boundary.

Proposition 3.1. Let $[M] \in \Omega_{n}^{U}(S F, F)$ and $\left\{F_{i}\right\}$ be the totality of connected components of the fixed point set of the circle action on $M$. If $D_{i}$ is an equivariant closed tubular neighborhood of $F_{i}$ with respect to an equivariant Hermitian metric on $M$, then we have

$$
[M]=\sum_{i}\left[D_{i}\right]
$$

in $\Omega_{n}^{U}(S F, F)$.

Proof. The result follows directly from $[4,5.2]$ because the circle action restricted to $M \backslash \cup_{i} D_{i}$ is free.

Let $M$ be a $U$-manifold with a semifree circle action which respects the $U$-structure and is free on the boundary, and $X_{i}$ a connected component of the fixed point set of the circle action. Then $X_{i}$ has a natural $U$-structure and the normal bundle $v\left(X_{i}\right)$ of $X_{i}$ in $M$ becomes a complex vector bundle on which $S^{1}$ acts by $U$-structure preserving 
isomorphisms. Moreover, there is a decomposition of the normal bundle $v\left(X_{i}\right)=v_{i}^{+} \oplus$ $v_{i}^{-}$such that $\lambda \in S^{1} \subset \mathbb{C}^{*}$ acts on the complex bundles $v_{i}^{+}$and $v_{i}^{-}$by multiplication with $\lambda$ and $\lambda^{-1}$, respectively (see, for instance, $[\mathbf{9}]$ ).

In what follows we will denote a generic element $[M, f]$ of $\Omega_{k}^{U}(B U(p) \times B U(q))$ by $[M, E \oplus G]$, where the complex bundles $E$ and $G$ of complex ranks $p$ and $q$ are the pullbacks of the universal bundles over the classifying spaces $B U(p)$ and $B U(q)$ under the first and second components of $f$, respectively. Consider the homomorphism

$$
\theta: \Omega_{n}^{U}(S F, F) \rightarrow \bigoplus_{k+2 p+2 q=n} \Omega_{k}^{U}(B U(p) \times B U(q))
$$

defined by

$$
\theta[M]=\sum_{i}\left[X_{i}, v_{i}^{+} \oplus v_{i}^{-}\right]
$$

where the summation is taken over the connected components of the fixed point set. It is a well-defined homomorphism.

Proposition 3.2. The homomorphism

$$
\theta: \Omega_{n}^{U}(S F, F) \rightarrow \bigoplus_{k+2 p+2 q=n} \Omega_{k}^{U}(B U(p) \times B U(q))
$$

is an isomorphism.

Proof. We will prove the result by exhibiting an inverse for $\theta$. Define the homomorphism

$$
\phi: \bigoplus_{k+2 p+2 q=n} \Omega_{k}^{U}(B U(p) \times B U(q)) \rightarrow \Omega_{n}^{U}(S F, F)
$$

by sending an element $[X, E \oplus G]$ of $\Omega_{k}^{U}(B U(p) \times B U(q))$ to $[D(E \oplus G)]$, where $E$ and $G$ are vector bundles over X of complex ranks $p$ and $q$ respectively, and $D(E \oplus G)$ is the disk bundle with respect to an equivariant metric on the vector bundle $E \oplus G$. It is a well-defined homomorphism. Clearly $\theta \circ \phi=$ identity and by Proposition 3.1 it follows that $\phi \circ \theta=$ identity. Thus $\theta$ is an isomorphism.

\subsection{The group $\Omega_{n}^{U, N}(S F, F)$}

This subsection is devoted to studying the group $\Omega_{n}^{U, N}(S F, F)$ of bordism classes of semifree circle actions on compact $n$-dimensional complex $N$-Spin manifolds which are free on the boundary. Let $\gamma_{p}$ and $\gamma_{q}$ be the universal complex vector bundles (of real ranks $2 p$ and $2 q$ ) over $B U(p)$ and $B U(q)$, respectively. We denote by $\Omega_{k}^{U, N}\left(B U(p) \times B U(q), \gamma_{p} \oplus \gamma_{q}\right)$ the bordism group consisting of 4-tuples $(M, f, g, s)$ such that $f: M \rightarrow B U(p)$ and $g: M \rightarrow B U(q)$ are continuous maps on a $(k-2 p-$ $2 q$ )-dimensional $U$-manifold $M$ and $s$ is a complex $N$-Spin structure on $\tau(M) \oplus$ $f^{*}\left(\gamma_{p}\right) \oplus g^{*}\left(\gamma_{q}\right)$. (Here by a complex $N$-Spin structure we mean a complex $N$-Spin structure on the stable bundle representing the $U$-structure.) The bordism relation between the elements of $\Omega_{k}^{U, N}\left(B U(p) \times B U(q), \gamma_{p} \oplus \gamma_{q}\right)$ is defined as follows: Two 4tuples $\left(M_{1}, f_{1}, g_{1}, s_{1}\right)$ and $\left(M_{2}, f_{2}, g_{2}, s_{2}\right)$ are said to be bordant if there is a third 4-tuple $(W, f, g, s)$ with a $(k-2 p-2 q+1)$-dimensional $U$-manifold $W$ such that $\partial W=M_{1} \cup-M_{2}, f\left|M_{1}=f_{1}, f\right| M_{2}=f_{2}, g\left|M_{1}=g_{1}, g\right| M_{2}=g_{2}$, and the complex $N$-Spin structures $s_{1}$ and $s_{2}$ are induced by $s$. 
Now we intend to show that the groups $\Omega_{n}^{U, N}(S F, F)$ are isomorphic to the groups $\bigoplus_{k+2 p+2 q=n} \Omega_{k}^{U, N}\left(B U(p) \times B U(q), \gamma_{p} \oplus \gamma_{q}\right)$. First we need to define a homomorphism

$$
\nu: \Omega_{n}^{U, N}(S F, F) \rightarrow \bigoplus_{k+2 p+2 q=n} \Omega_{k}^{U, N}\left(B U(p) \times B U(q), \gamma_{p} \oplus \gamma_{q}\right) .
$$

Let $[M, \alpha]$ be an element of $\Omega_{n}^{U, N}(S F, F)$ with the given complex $N$-Spin structure on $M$ denoted by $s$ and $X^{k-2 p-2 q}$ the union of the $(k-2 p-2 q)$-dimensional components of the fixed point set of the circle action $\alpha$. Also, denote by $v_{p, q}$ the normal bundle of $X^{k-2 p-2 q}$ in $M$. The complex $N$-Spin structure $s$ on $M$ induces a complex $N$-Spin structure $s_{p, q}$ on $\tau\left(X^{k-2 p-2 q}\right) \oplus v_{p, q}$ because $\tau(M) \mid X^{k-2 p-2 q} \cong \tau\left(X^{k-2 p-2 q}\right) \oplus v_{p, q}$. Now define

$$
\nu: \Omega_{n}^{U, N}(S F, F) \rightarrow \bigoplus_{k+2 p+2 q=n} \Omega_{k}^{U, N}\left(B U(p) \times B U(q), \gamma_{p} \oplus \gamma_{q}\right)
$$

by

$$
\nu([M, \alpha])=\bigoplus_{k+2 p+2 q=n}\left[X^{k-2 p-2 q}, f_{p, q}, g_{p, q}, s_{p, q}\right],
$$

where $f_{p, q}$ and $g_{p, q}$ are the classifying maps of $v_{p, q}^{+}$and $v_{p, q}^{-}$, respectively. It is a routine matter to see that $\nu$ is a well-defined homomorphism.

Proposition 3.3. The homomorphism

$$
\nu: \Omega_{n}^{U, N}(S F, F) \rightarrow \bigoplus_{k+2 p+2 q=n} \Omega_{k}^{U, N}\left(B U(p) \times B U(q), \gamma_{p} \oplus \gamma_{q}\right)
$$

is an isomorphism.

Proof. We will prove the result by showing that the homomorphism $\nu$ has an inverse. Let $[M, f, g, s] \in \Omega_{k}^{U, N}\left(B U(p) \times B U(q), \gamma_{p} \oplus \gamma_{q}\right)$ and set $E:=f^{*}\left(\gamma_{p}\right), G:=g^{*}\left(\gamma_{q}\right)$, then $f$ and $g$ give rise to the maps $\hat{f}: D(E) \rightarrow D\left(\gamma_{p}\right)$ and $\hat{g}: D(G) \rightarrow D\left(\gamma_{q}\right)$ between the disk bundles which may be assumed transverse regular to the zero sections $B U(p)$ and $B U(q)$ of $\gamma_{p}$ and $\gamma_{q}$, respectively. So one gets $v(M, E)=f^{*}\left(v\left(B U(p), \gamma_{p}\right)\right)=$ $f^{*}\left(\gamma_{p}\right)=E$ and $v(M, G)=g^{*}\left(v\left(B U(q), \gamma_{q}\right)\right)=g^{*}\left(\gamma_{q}\right)=G$, where $v$ denotes normal bundle. If $\pi: E \oplus G \rightarrow M$ denotes the projection, then by using the identity $\tau(E \oplus$ $G)=\pi^{*}(\tau(M) \oplus E \oplus G)$ we have that the complex $N$-Spin structure $s$ induces a complex $N$-Spin structure $\pi^{*}(s)$ on $\tau(E \oplus G)$ which restricts to a complex $N$-Spin structure on the disk bundle $D(E \oplus G)$. If we denote by $\beta$ the complex multiplication on the bundle $E$ and complex multiplication after inverse on $G$, then the assignment $[M, f, g, s] \rightarrow[D(E \oplus G), \beta]$ gives an inverse of $\nu$.

From now on we will denote a generic element $[M, f, g, s]$ of $\Omega_{k}^{U, N}(B U(p) \times B U(q)$, $\left.\gamma_{p} \oplus \gamma_{q}\right)$ simply by $[M, h, s]$, where $h$ is the map $(f, g): M \rightarrow B U(p) \times B U(q)$. The bordism group $\Omega_{k}^{U}\left(B U(p) \times B U(q), \gamma_{p} \oplus \gamma_{q}\right)$ can be defined similar to the bordism group $\Omega_{k}^{U, N}\left(B U(p) \times B U(q), \gamma_{p} \oplus \gamma_{q}\right)$ by considering only a $U$-structure in place of a complex $N$-Spin structure, and the following result can be proved along the lines of Proposition 3.3 . 
Proposition 3.4. The homomorphism

$$
\nu^{\prime}: \Omega_{n}^{U}(S F, F) \rightarrow \bigoplus_{k+2 p+2 q=n} \Omega_{k}^{U}\left(B U(p) \times B U(q), \gamma_{p} \oplus \gamma_{q}\right)
$$

is an isomorphism, where $\nu^{\prime}$ has similar meaning as $\nu$.

Next, we want to show that the bordism group $\Omega_{k}^{U, N}\left(B U(p) \times B U(q), \gamma_{p} \oplus \gamma_{q}\right)$ is isomorphic to the complex $N$-Spin bordism group $\Omega_{k}^{U, N}\left(D\left(\gamma_{p} \oplus \gamma_{q}\right), S\left(\gamma_{p} \oplus \gamma_{q}\right)\right)$ of pair $\left(D\left(\gamma_{p} \oplus \gamma_{q}\right), S\left(\gamma_{p} \oplus \gamma_{q}\right)\right)$, where $D\left(\gamma_{p} \oplus \gamma_{q}\right)$ and $S\left(\gamma_{p} \oplus \gamma_{q}\right)$ denote the disk and sphere bundles of $\gamma_{p} \oplus \gamma_{q}$, respectively. Define

$$
\rho: \Omega_{k}^{U, N}\left(B U(p) \times B U(q), \gamma_{p} \oplus \gamma_{q}\right) \rightarrow \Omega_{k}^{U, N}\left(D\left(\gamma_{p} \oplus \gamma_{q}\right), S\left(\gamma_{p} \oplus \gamma_{q}\right)\right)
$$

by

$$
\rho[M, h, s]=\left[D\left(h^{*}\left(\gamma_{p} \oplus \gamma_{q}\right)\right), \hat{h}\left|D\left(h^{*}\left(\gamma_{p} \oplus \gamma_{q}\right)\right), \pi^{*}(s)\right| D\left(h^{*}\left(\gamma_{p} \oplus \gamma_{q}\right)\right)\right],
$$

where $\hat{h}$ is the map from $h^{*}\left(\gamma_{p} \oplus \gamma_{q}\right)$ to $\gamma_{p} \oplus \gamma_{q}$ induced by $h$. Since $\tau\left(h^{*}\left(\gamma_{p} \oplus \gamma_{q}\right)\right)=$ $\pi^{*}\left(\tau(M) \oplus h^{*}\left(\gamma_{p} \oplus \gamma_{q}\right)\right)$, where $\pi: h^{*}\left(\gamma_{p} \oplus \gamma_{q}\right) \rightarrow M$ is the projection, the complex $N$-Spin structure $s$ induces a complex $N$-Spin structure $\pi^{*}(s)$ on $h^{*}\left(\gamma_{p} \oplus \gamma_{q}\right)$. It is clear that $\rho$ is a well-defined homomorphism.

Proposition 3.5. The homomorphism

$$
\rho: \Omega_{k}^{U, N}\left(B U(p) \times B U(q), \gamma_{p} \oplus \gamma_{q}\right) \rightarrow \Omega_{k}^{U, N}\left(D\left(\gamma_{p} \oplus \gamma_{q}\right), S\left(\gamma_{p} \oplus \gamma_{q}\right)\right)
$$

is an isomorphism.

Proof. We will prove the result by exhibiting an inverse for $\rho$. A generic element of $\Omega_{k}^{U, N}\left(D\left(\gamma_{p} \oplus \gamma_{q}\right), S\left(\gamma_{p} \oplus \gamma_{q}\right)\right)$ is represented by a bordism class $[W, H, s]$, where $W$ is a $k$-dimensional compact complex $N$-Spin manifold with the given complex $N$-Spin structure $s$ and $H$ is a continuous map $(W, \partial W) \rightarrow\left(D\left(\gamma_{p} \oplus \gamma_{q}\right), S\left(\gamma_{p} \oplus \gamma_{q}\right)\right)$. One may choose $H$ so that it is transverse regular to the zero section $B U(p) \times B U(q)$ of $\gamma_{p} \oplus$ $\gamma_{q}$. Put $M:=H^{-1}(B U(p) \times B U(q))$ and $h:=H \mid M$. Since $v(M, W)=h^{*}(v(B U(p) \times$ $\left.\left.B U(q), \gamma_{p} \oplus \gamma_{q}\right)\right)=h^{*}\left(\gamma_{p} \oplus \gamma_{q}\right)$ and $\tau(W) \mid M=\tau(M) \oplus v(M, W)$, therefore $\tau(W) \mid M$ $=\tau(M) \oplus h^{*}\left(\gamma_{p} \oplus \gamma_{q}\right)$. It follows that the restriction of $s$ induces a complex $N$-Spin structure $\hat{s}$ on $\tau(M) \oplus h^{*}\left(\gamma_{p} \oplus \gamma_{q}\right)$. Now define

$$
\delta: \Omega_{k}^{U, N}\left(D\left(\gamma_{p} \oplus \gamma_{q}\right), S\left(\gamma_{p} \oplus \gamma_{q}\right)\right) \rightarrow \Omega_{k}^{U, N}\left(B U(p) \times B U(q), \gamma_{p} \oplus \gamma_{q}\right)
$$

by

$$
\delta[W, H, s]=[M, h, \hat{s}] .
$$

Clearly $\delta$ is a homomorphism and $\delta \circ \rho=$ identity. We need to prove that $\rho \circ \delta=$ identity. Observe first that

$$
\begin{aligned}
\rho \circ \delta[W, H, s] & =\rho[M, h, \hat{s}] \\
& =\left[D\left(h^{*}\left(\gamma_{p} \oplus \gamma_{q}\right)\right), \hat{h}\left|D\left(h^{*}\left(\gamma_{p} \oplus \gamma_{q}\right)\right), \pi^{*}(\hat{s})\right| D\left(h^{*}\left(\gamma_{p} \oplus \gamma_{q}\right)\right)\right] .
\end{aligned}
$$

Now we must show that $\left(D\left(h^{*}\left(\gamma_{p} \oplus \gamma_{q}\right)\right), \hat{h}\left|D\left(h^{*}\left(\gamma_{p} \oplus \gamma_{q}\right)\right), \pi^{*}(\hat{s})\right| D\left(h^{*}\left(\gamma_{p} \oplus \gamma_{q}\right)\right)\right)$ and $(W, H, s)$ represent the same bordism class in $\Omega_{k}^{U, N}\left(D\left(\gamma_{p} \oplus \gamma_{q}\right), S\left(\gamma_{p} \oplus \gamma_{q}\right)\right)$ by constructing a bordism between them. The unit interval $I=[0,1]$ has a natural complex $N$-Spin structure corresponding to a stable $U$-structure (see, for instance, [9]). 
We identify $D\left(h^{*}\left(\gamma_{p} \oplus \gamma_{q}\right)\right)$ with a tubular neighborhood of $M$ in $W$. Then, after smoothing a corner at $W \times 1$ and introducing a corner at $S\left(h^{*}\left(\gamma_{p} \oplus \gamma_{q}\right)\right) \times 1$, one can interpret $(W \times I, h, s)$ as a bordism between $(W, H, s)$ and $\left(D\left(h^{*}\left(\gamma_{p} \oplus \gamma_{q}\right)\right)\right.$, $\left.H\left|D\left(h^{*}\left(\gamma_{p} \oplus \gamma_{q}\right)\right), s\right| D\left(h^{*}\left(\gamma_{p} \oplus \gamma_{q}\right)\right)\right)$. The maps $\hat{h}$ and

$$
H:\left(D\left(h^{*}\left(\gamma_{p} \oplus \gamma_{q}\right)\right), S\left(h^{*}\left(\gamma_{p} \oplus \gamma_{q}\right)\right)\right) \rightarrow\left(D\left(\gamma_{p} \oplus \gamma_{q}\right), S\left(\gamma_{p} \oplus \gamma_{q}\right)\right)
$$

are homotopic, so $\rho \circ \delta[W, H, s]=[W, H, s]$ and this finishes the proof.

The following result can be proved along the lines of Proposition 3.5.

Proposition 3.6. The homomorphism

$$
\rho^{\prime}: \Omega_{k}^{U}\left(B U(p) \times B U(q), \gamma_{p} \oplus \gamma_{q}\right) \rightarrow \Omega_{k}^{U}\left(D\left(\gamma_{p} \oplus \gamma_{q}\right), S\left(\gamma_{p} \oplus \gamma_{q}\right)\right)
$$

is an isomorphism, where $\rho^{\prime}$ has similar meaning as $\rho$.

We conclude this subsection by combining Proposition 3.3 with Proposition 3.5 and Proposition 3.4 with Proposition 3.6 into the following two results.

Proposition 3.7. We have an isomorphism:

$$
\rho \circ \nu: \Omega_{n}^{U, N}(S F, F) \rightarrow \bigoplus_{k+2 p+2 q=n} \Omega_{k}^{U, N}\left(D\left(\gamma_{p} \oplus \gamma_{q}\right), S\left(\gamma_{p} \oplus \gamma_{q}\right)\right) .
$$

Proposition 3.8. We have an isomorphism:

$$
\rho^{\prime} \circ \nu^{\prime}: \Omega_{n}^{U}(S F, F) \rightarrow \bigoplus_{k+2 p+2 q=n} \Omega_{k}^{U}\left(D\left(\gamma_{p} \oplus \gamma_{q}\right), S\left(\gamma_{p} \oplus \gamma_{q}\right)\right) .
$$

Since a complex $N$-Spin bordism in the groups $\Omega_{n}^{U, N}(S F, F), \Omega_{k}^{U, N}(B U(p) \times B U(q)$, $\left.\gamma_{p} \oplus \gamma_{q}\right)$, and $\Omega_{k}^{U, N}\left(D\left(\gamma_{p} \oplus \gamma_{q}\right), S\left(\gamma_{p} \oplus \gamma_{q}\right)\right)$ is also a $U$-bordism in the groups $\Omega_{n}^{U}(S F$, $F), \Omega_{k}^{U, N}\left(B U(p) \times B U(q), \gamma_{p} \oplus \gamma_{q}\right)$, and $\Omega_{k}^{U}\left(D\left(\gamma_{p} \oplus \gamma_{q}\right), S\left(\gamma_{p} \oplus \gamma_{q}\right)\right)$, respectively, therefore the forgetful homomorphisms

$$
\begin{aligned}
\Omega_{n}^{U, N}(S F, F) & \rightarrow \Omega_{n}^{U}(S F, F), \\
\Omega_{k}^{U, N}\left(B U(p) \times B U(q), \gamma_{p} \oplus \gamma_{q}\right) & \rightarrow \Omega_{k}^{U}\left(B U(p) \times B U(q), \gamma_{p} \oplus \gamma_{q}\right),
\end{aligned}
$$

and

$$
\Omega_{k}^{U, N}\left(D\left(\gamma_{p} \oplus \gamma_{q}\right), S\left(\gamma_{p} \oplus \gamma_{q}\right)\right) \rightarrow \Omega_{k}^{U}\left(D\left(\gamma_{p} \oplus \gamma_{q}\right), S\left(\gamma_{p} \oplus \gamma_{q}\right)\right)
$$

are well-defined.

Proposition 3.9. The diagrams

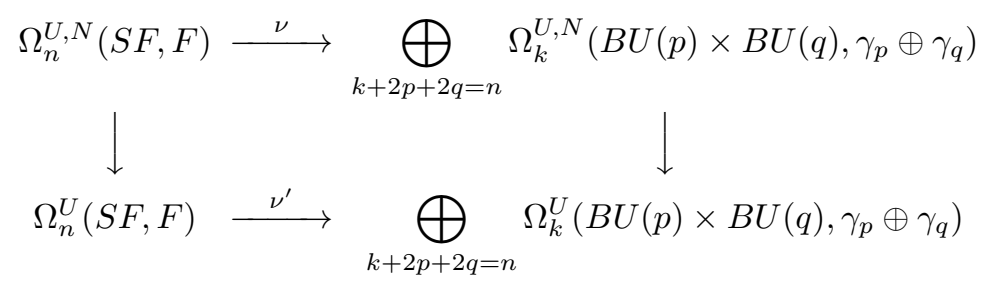


and

$$
\begin{gathered}
\bigoplus_{k+2 p+2 q=n} \Omega_{k}^{U, N}\left(B U(p) \times B U(q), \gamma_{p} \oplus \gamma_{q}\right) \stackrel{\rho}{\longrightarrow} \underset{k+2 p+2 q=n}{\bigoplus_{k} \Omega_{k}^{U, N}\left(D\left(\gamma_{p} \oplus \gamma_{q}\right), S\left(\gamma_{p} \oplus \gamma_{q}\right)\right)} \\
\bigoplus_{k+2 p+2 q=n} \Omega_{k}^{U}\left(B U(p) \times B U(q), \gamma_{p} \oplus \gamma_{q}\right) \stackrel{\rho^{\prime}}{\longrightarrow} \bigoplus_{k+2 p+2 q=n} \Omega_{k}^{U}\left(D\left(\gamma_{p} \oplus \gamma_{q}\right), S\left(\gamma_{p} \oplus \gamma_{q}\right)\right)
\end{gathered}
$$

are commutative, where vertical arrows are the forgetful homomorphisms.

Proof. Note that $\nu^{\prime}$ and $\rho^{\prime}$ have the definitions similar to $\nu$ and $\rho$, respectively, and the vertical arrows are forgetful homomorphisms. Therefore, the result follows by using the definitions of maps involved in the diagram.

\subsection{The group $\Omega_{n}^{U, N, t}(S F, F) \otimes \mathbb{Q}$}

In this subsection we will tensor bordism groups considered in the previous two subsections with the rationals $\mathbb{Q}$. It will give us more traction on those groups and will help by using the results of those sections to compute the group $\Omega_{n}^{U, N}(S F, F) \otimes \mathbb{Q}$, and then the group $\Omega_{n}^{U, N, t}(S F, F) \otimes \mathbb{Q}$ which appears in the exact sequence (3.1).

It is a routine matter to check that $\Omega_{K}^{U, N}(\cdot)$ is a generalized homology theory (see, for instance, $[\mathbf{3}$, p. 9]). Hence it becomes trivial when tensored with the rationals (see, for instance, $[\mathbf{6}]$ ). Since the forgetful map $\Omega_{*}^{U, N} \rightarrow \Omega_{*}^{U}$ is a rational isomorphism (see $[\mathbf{9}]$ ), therefore, we have the following result:

Proposition 3.10. Let $(X, A)$ be a finite $C W$-pair. The forgetful homomorphism

$$
\Omega_{n}^{U, N}(X, A) \otimes \mathbb{Q} \rightarrow \Omega_{n}^{U}(X, A) \otimes \mathbb{Q}
$$

is an isomorphism.

Lemma 3.11. The forgetful homomorphism $\Omega_{n}^{U, N}(S F, F) \otimes \mathbb{Q} \rightarrow \Omega_{n}^{U}(S F, F) \otimes \mathbb{Q}$ is an isomorphism.

Proof. The diagrams in Proposition 3.9 remain commutative after tensoring with the rationals $\mathbb{Q}$. Putting them together after tensoring with $\mathbb{Q}$, we get the commutative diagram

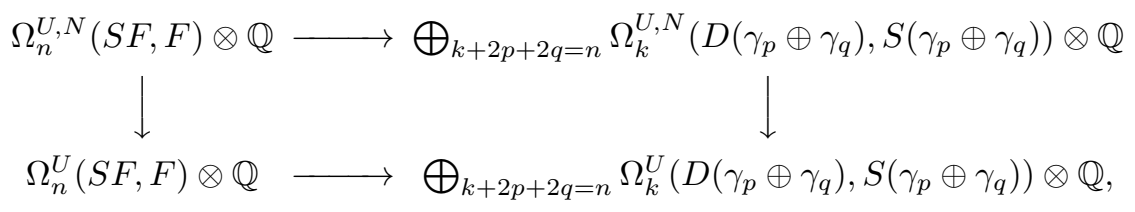

where vertical arrows are the forgetful homomorphisms and horizontal arrows are the composites of the isomorphisms from Proposition 3.9, tensored with the rationals $\mathbb{Q}$. Since the right hand vertical arrow is an isomorphism by Proposition 3.10, it follows that the left hand vertical arrow is also an isomorphism.

Now the following result gives a way to compute the group $\Omega_{n}^{U, N}(S F, F) \otimes \mathbb{Q}$. 
Lemma 3.12. We have an isomorphism

with $\vartheta$ given by

$$
\vartheta \otimes \mathbb{Q}: \Omega_{n}^{U, N}(S F, F) \otimes \mathbb{Q} \rightarrow \bigoplus_{k+2 p+2 q=n} \Omega_{k}^{U}(B U(p) \times B U(q)) \otimes \mathbb{Q},
$$

$$
\vartheta[M]=\sum_{i}\left[X_{i}, v_{i}^{+} \oplus v_{i}^{-}\right]
$$

where the summation is taken over the connected components of the fixed point set, and $v_{i}^{+}, v_{i}^{-}$have the same meanings as in Proposition 3.2.

Proof. The result follows from Lemma 3.11 and Proposition 3.2.

Note that we have the decompositions

$$
\Omega_{n}^{U, N}(S F, F) \otimes \mathbb{Q}=\bigoplus_{t \in \mathbb{Z} / N \mathbb{Z}} \Omega_{n}^{U, N, t}(S F, F) \otimes \mathbb{Q}
$$

and

$$
\bigoplus_{k+2 p+2 q=n} \Omega_{k}^{U}(B U(p) \times B U(q)) \otimes \mathbb{Q}=\bigoplus_{\substack{k+2 p+2 q=n \\ p-q \equiv t(\bmod N)}} \Omega_{k}^{U}(B U(p) \times B U(q)) \otimes \mathbb{Q} .
$$

The type of an $S^{1}$-action on a complex $N$-Spin manifold is the sum of rotation numbers of a fixed point component modulo $N$. Hence the summands involving $t$ in above two decompositions correspond to each other under the isomorphism of groups in Lemma 3.12. Thus we have:

Theorem 3.13. The homomorphism

$$
\Omega_{n}^{U, N, t}(S F, F) \otimes \mathbb{Q} \rightarrow \bigoplus_{\substack{k+2 p+2 q=n \\ p-q \equiv t(\bmod N)}} \Omega_{k}^{U}(B U(p) \times B U(q)) \otimes \mathbb{Q}
$$

given by

$$
[M] \mapsto \sum_{i}\left[X_{i}, v_{i}^{+} \oplus v_{i}^{-}\right]
$$

is an isomorphism.

\subsection{Complex $N$-Spin ${ }^{c, t}$ structures}

Let $N$ and $t$ be two positive integers such that $t \mid N$. In this subsection we introduce the notion of a complex $N$-Spin ${ }^{c, t}$ structure which is needed to characterize the rational complex $N$-Spin bordism group having a free circle action of type $t$. In what follows we will use the identifications $U(1) \cong S^{1} \cong \mathbb{R} / \mathbb{Z}$ and $\hat{U}(1) \cong \mathbb{R} / N \mathbb{Z} \cong S^{1}$ without further mention. At times we will also use $S^{1}$ and $U(1)$ separately when it is needed to distinguish between the actions on manifolds and bundles, respectively.

Let $\left(\mathbf{Z}_{N}, \cdot\right)$ denote the group of $N$-th roots of unity generated by $e^{\frac{2 \pi i}{N}}$. It is isomorphic to the group $\left(\mathbb{Z}_{N},+\right)$ of residue classes of integers $\bmod N$. So we note for a later use that one can identify the cohomology groups $H^{n}\left(X ; \mathbf{Z}_{N}\right)$ and $H^{n}\left(X ; \mathbb{Z}_{N}\right)$ for any space $X$ and any nonnegative integer $n$. Let $\lambda_{N}: \hat{U}(1) \rightarrow U(1)$ given by $\lambda \mapsto \lambda^{N}$ be the $N$-fold covering of $U(1)$. Note that $\mathbf{Z}_{N}=\operatorname{ker} \lambda_{N}$. We denote by $i: \mathbf{Z}_{N} \rightarrow \hat{U}(1)$ the inclusion. 
Definition 3.14. Let $N$ and $t$ be positive integers such that $t \mid N$, and let $\mu_{N / t}: \hat{S}^{1} \rightarrow$ $S^{1}$ given by $\zeta \mapsto \zeta^{N / t}$ be the $N / t$-fold covering of $S^{1}$. Let $\mathbf{Z}_{N}$ act on $\hat{U}(1) \times \hat{S}^{1}$ by sending $(\lambda, g, \zeta)$ to $\left(\lambda g, \lambda^{-t} \zeta\right)$, where $\lambda \in \mathbf{Z}_{N}, g \in \hat{U}(1)$, and $\zeta \in \hat{S}^{1}$. We define $U^{N, c, t}$ as the group $\hat{U}(1) \underset{\mathbf{z}_{N}}{\times} \hat{S}^{1}$.

We will represent a generic element of $U^{N, c, t}$ by $[g, \zeta]$, where $g \in \hat{U}(1)$, and $\zeta \in \hat{S}^{1}$. Notice that $\mathbf{Z}_{N / t}$ can be considered as a subgroup of $U^{N, c, t}$ generated by $\left[e^{\frac{2 \pi i}{N}}, 1\right]=$ $\left[\left(e^{\frac{2 \pi i}{N}}\right)^{-1} e^{\frac{2 \pi i}{N}},\left(\left(e^{\frac{2 \pi i}{N}}\right)^{-1}\right)^{-t} 1\right]=\left[1, e^{\frac{2 \pi t i}{N}}\right]$. Let $\mu: U^{N, c, t} \rightarrow U(1) \times S^{1}$ be induced by the map $\lambda_{N} \times \mu_{N / t}: \hat{U}(1) \times \hat{S}^{1} \rightarrow U(1) \times S^{1}$. Then we have a short exact sequence:

$$
0 \longrightarrow \mathbf{Z}_{N / t} \longrightarrow U^{N, c, t} \stackrel{\mu}{\longrightarrow} U(1) \times S^{1} \longrightarrow 1 \text {. }
$$

Definition 3.15. Let $\Delta$ be a principal $U(1)$-bundle over a finite connected $C W$ complex B. A complex $N$-Spin ${ }^{c, t}$ structure (or simply an $N^{c, t}$-structure) on $\Delta$ consists of

1. a principal $U^{N, c, t}$-bundle $\eta$ over $B$,

2. a principal $U(1)$-bundle $\Lambda$ over $B$, and

3. a bundle map $f: E(\eta) \rightarrow E(\Delta) \times E(\Lambda)$ such that $f(x g)=f(x) \mu(g)$, where $x \in$ $E(\eta)$, and $g \in U^{N, c, t}$.

Let $\xi$ be a principal $U(n)$-bundle over a finite connected $C W$-complex $B$ and $\Delta$ be the associated principal bundle belonging to the determinant representation $U(n) \rightarrow U(1)$. A complex $N$-Spin ${ }^{c, t}$ structure (or simply an $N^{c, t}$-structure) on $\xi$ is a complex $N$-Spin ${ }^{c, t}$ structure on $\Delta$. The notion of a complex $N$-Spin ${ }^{c, t}$ structure is a generalization of the notion of a $\operatorname{Spin}^{c}$ structure, and a complex $N$-Spin ${ }^{c, t}$ structure becomes a complex $\operatorname{Spin}^{c}$ structure if $N=2$ and $t=1$. We will see that every principal $U(n)$-bundle over a finite $C W$-complex admits a complex $N$-Spin ${ }^{c, 1}$ structure. The coefficient sequence (3.2) determines the exact sequence:

$$
H^{1}\left(B ; U^{N, c, t}\right) \stackrel{\mu_{*}}{\longrightarrow} H^{1}(B ; U(1)) \oplus H^{1}\left(B ; S^{1}\right) \stackrel{\hat{c}_{N / t}}{\longrightarrow} H^{2}\left(B ; \mathbf{Z}_{N / t}\right) .
$$

The map $\hat{c}_{N / t}$ sends a pair $(\Delta, \Lambda)$ to the cohomology class $\rho_{N / t}\left(c_{1}(\Delta)\right)-t \rho_{N / t}\left(c_{1}(\Lambda)\right)$, where $\rho_{N / t}: H^{2}(B ; \mathbb{Z}) \rightarrow H^{2}\left(B ; \mathbf{Z}_{N / t}\right)$ is the $\bmod N / t$ reduction. It follows by the exactness of sequence (3.3) that the bundle $\xi$ (equivalently $\Delta$ ) can be given a complex $N$-Spin ${ }^{c, t}$ structure if and only if there exists a cohomology class $u$ in $H^{2}(B ; \mathbb{Z})$ such that $\rho_{N / t}\left(c_{1}(\Delta)\right)=t \rho_{N / t}(u)$. Since the first Chern class $c_{1}(\xi)$ of $\xi$ is the same as the first Chern class $c_{1}(\Delta)$ of the determinant line bundle $\Delta$, one can take $u=c_{1}(\xi)$ to see that the principal $U(n)$-bundle $\xi$ admits a complex $N$-Spin ${ }^{c, 1}$ structure.

Proposition 3.16. Let $E_{1}$ and $E_{2}$ be two complex vector bundles over a finite $C W$ complex B. Then a choice of complex $N$-Spin ${ }^{c, t}$ structures on two of the three bundles $E_{1}, E_{2}, E_{1} \oplus E_{2}$ uniquely determines a complex $N$-Spin ${ }^{c, t}$ structure on the third one.

Proof. The bundles $E_{1}$ and $E_{2}$ can be provided with Hermitian metrics to look at the associated principal $U(n)$-bundles. Let $u_{1}, u_{2} \in H^{2}(B ; \mathbb{Z})$ be two cohomology classes such that $\rho_{N / t}\left(c_{1}\left(E_{1}\right)\right)=t \rho_{N / t}\left(u_{1}\right)$ and $\rho_{N / t}\left(c_{1}\left(E_{2}\right)\right)=t \rho_{N / t}\left(u_{2}\right)$. Since $c_{1}\left(E_{1} \oplus E_{2}\right)=c_{1}\left(E_{1}\right)+c_{2}\left(E_{2}\right)$, therefore, $\rho_{N / t}\left(c_{1}\left(E_{1} \oplus E_{2}\right)\right)=\rho_{N / t}\left(c_{1}\left(E_{1}\right)+c_{2}\left(E_{2}\right)\right)$ $=\rho_{N / t}\left(c_{1}\left(E_{1}\right)\right)+\rho_{N / t}\left(c_{2}\left(E_{2}\right)\right)$, and so $\rho_{N / t}\left(c_{1}\left(E_{1} \oplus E_{2}\right)\right)=t \rho_{N / t}\left(u_{1}\right)+t \rho_{N / t}\left(u_{2}\right)=$ $t \rho_{N / t}\left(u_{1}+u_{2}\right)$ with $u_{1}+u_{2} \in H^{2}(B ; \mathbb{Z})$. Hence a choice of complex $N$-Spin ${ }^{c, t}$ struc- 
tures on $E_{1}$ and $E_{2}$ uniquely determines a complex $N$-Spin ${ }^{c, t}$ structure on $E_{1} \oplus$ $E_{2}$. Similarly, the equations $\rho_{N / t}\left(c_{1}\left(E_{1}\right)\right)=\rho_{N / t}\left(c_{1}\left(E_{1} \oplus E_{2}\right)\right)-\rho_{N / t}\left(c_{1}\left(E_{2}\right)\right)$ and $\rho_{N / t}\left(c_{1}\left(E_{2}\right)\right)=\rho_{N / t}\left(c_{1}\left(E_{1} \oplus E_{2}\right)\right)-\rho_{N / t}\left(c_{1}\left(E_{1}\right)\right)$ can be used to prove the other two cases.

\subsection{The group $\Omega_{n}^{U, N^{c, t}}$}

We define a complex $N$-Spin ${ }^{c, t}$ manifold (or simply an $N^{c, t}$-manifold) as a $U$ manifold together with a complex $N$-Spin ${ }^{c, t}$ structure on the principal $U(n)$-bundle belonging to its stable tangent bundle representing its $U$-structure. We will denote the cobordism group of $n$-dimensional connected closed complex $N$-Spin ${ }^{c, t}$ manifolds by $\Omega_{n}^{U, N^{c, t}}$.

Let $\psi: B \rightarrow B U(n)$ be a classifying map of a $U(n)$-bundle $\xi$ and consider the map $\bar{c}_{1}-t \bar{c}_{1}: B S^{1} \times K(\mathbb{Z}, 2) \rightarrow K\left(\mathbb{Z}_{N / t}, 2\right)$, where $\bar{c}_{1}$ is the first Chern class reduction $\bmod N / t$. Let $\phi_{N / t}$ be the pullback, with the map $\bar{c}_{1}-t \bar{c}_{1}$, of the fibration $p_{N / t}: P K\left(\mathbb{Z}_{N / t}, 2\right) \rightarrow K\left(\mathbb{Z}_{N / t}, 2\right)$ having the fiber homotopy type $\Omega K\left(\mathbb{Z}_{N / t}, 2\right) \cong$ $K\left(\mathbb{Z}_{N / t}, 1\right)$. Further pulling back the fibration $\phi_{N / t}$ with det $\times$ id to $B U(n) \times K(\mathbb{Z}, 2)$ gives the fibration $\left(\hat{\phi}_{N / t}\right)_{n}: \widehat{B U}(n) \rightarrow B U(n) \times K(\mathbb{Z}, 2)$ :

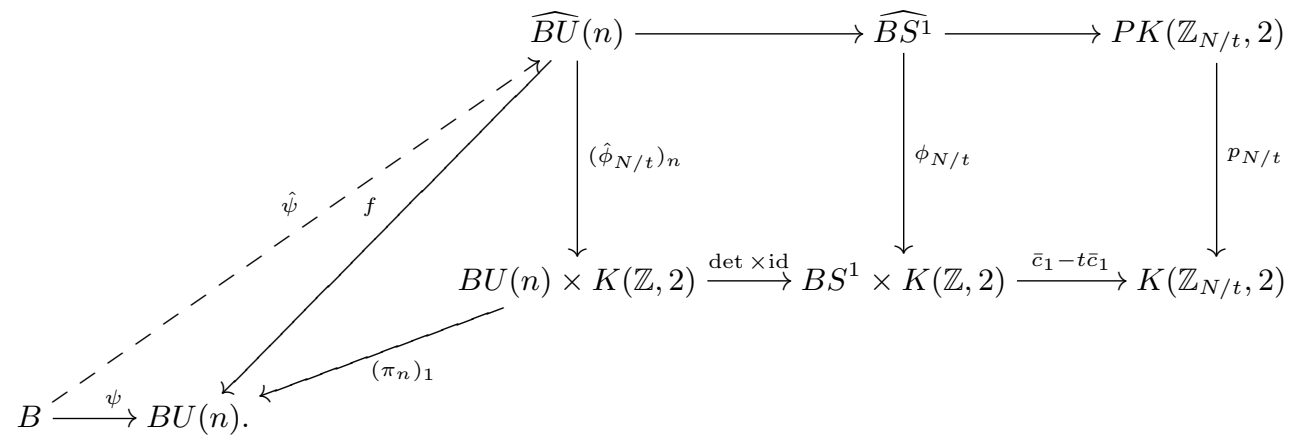

An equivalence class of a complex $N$-Spin ${ }^{c, t}$ structure on $\xi$ corresponds to a fiber homotopy class of a lifting $\hat{\psi}$ of $\psi$ regarded as fibration, where $f=\left(\pi_{n}\right)_{1} \circ\left(\hat{\phi}_{N / t}\right)_{n}$ and $\left(\pi_{n}\right)_{1}: B U(n) \times K(\mathbb{Z}, 2) \rightarrow B U(n)$ is the projection to the first component. The fibrations $\left(\hat{\phi}_{N / t}\right)_{n}: \widehat{B U}(n) \rightarrow B U(n) \times K(\mathbb{Z}, 2)$ induce the fibration $\hat{\phi}_{N / t}: \widehat{B U} \rightarrow B U$ over the stable classifying space:

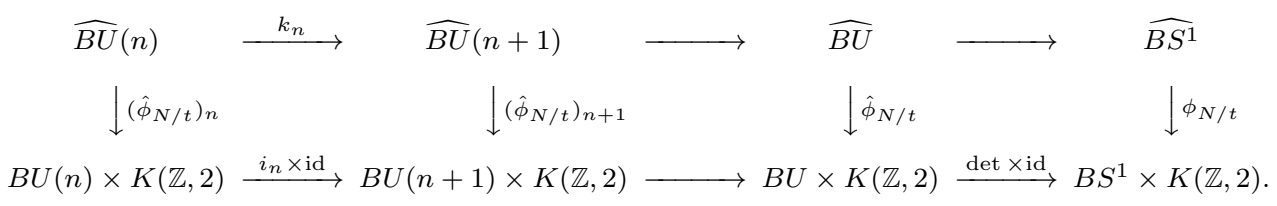

If one sets $B_{2 n}:=B_{2 n+1}:=\widehat{B U}(n)$, then one gets the following commutative diagram:

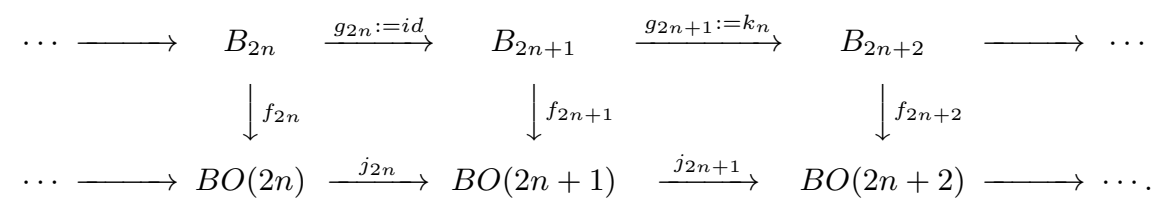

Here $j_{k}: B O(k) \rightarrow B O(k+1)$ is the natural map which belongs to the addition of 
a trivial real line bundle, and $f_{k}$ is the fibration defined by $f_{2 n}:=h_{n} \circ\left(\hat{\phi}_{N / t}\right)_{n}$, $f_{2 n+1}:=j_{2 n} \circ f_{2 n}$, where $h_{n}=q_{n} \circ\left(\pi_{n}\right)_{1}$ and $q_{n}: B U(n) \rightarrow B O(2 n)$ is the natural map belonging to "forgetting" the complex structure.

Theorem 3.17. The cobordism theory $\Omega_{n}(B, f)$, belonging to the above family of fibrations $f_{k}: B_{k} \rightarrow B O(k)$, corresponds to the cobordism group $\Omega_{n}^{U, N^{c, t}}$.

Proof. A complex $N$-Spin ${ }^{c, t}$ manifold $M$ with a given complex $N$-Spin ${ }^{c, t}$ structure corresponds to a unique stable equivalence class of the homotopy classes of the liftings $f_{l}: B_{l} \rightarrow B O(l)$ to the classifying maps of the normal bundle of $M$.

A complex $N$-Spin ${ }^{c, t}$ structure on the stable tangent bundle induces a complex $N$-Spin ${ }^{c, t}$-structure on the normal bundle: Embed $M$ differentiably in $\mathbb{R}^{2 s}, s$ large, then $\left(\tau(M) \oplus \epsilon_{\mathbb{R}}^{k}\right) \oplus \nu \cong \epsilon_{\mathbb{R}}^{2 s}$. Provide the tangent bundle $\epsilon_{\mathbb{R}}^{2 s}$ of $\mathbb{R}^{2 s}$ with the canonical $U$ - and complex $N$-Spin ${ }^{c, t}$ structures and restrict them to $M$. The normal bundle $\nu$ can be provided with a $U$-structure (see [5, p. 21]) and by Proposition 3.16 one gets the complex $N$-Spin ${ }^{c, t}$ structure. The relationship between $U$-structures and liftings is clear, we have discussed the relationship between complex $N$-Spin ${ }^{c, t}$ structures and liftings previously.

The set of equivalence classes $\Omega_{n}(B, f)$ corresponds to the set of equivalence classes $\Omega_{n}^{U, N^{c, t}}$.

Let $M$ be a complex $N$-Spin ${ }^{c, t}$ manifold. Let $f: M \rightarrow \mathbb{C P}^{\infty}$ denote the classifying map of the principal $U(1)$-bundle over $M$ which exists due to the second axiom of definition of complex $N$-Spin ${ }^{c, t}$ structure. We call the map $\Omega_{n}^{U, N^{c, t}} \rightarrow \Omega_{n}^{U}\left(\mathbb{C P}^{\infty}\right)$ which sends $[M]$ to $[M, f]$ the forgetful homomorphism.

Theorem 3.18. For $t \mid N$, the forgetful homomorphism

$$
\Omega_{n}^{U, N^{c, t}} \rightarrow \Omega_{n}^{U}\left(\mathbb{C P}^{\infty}\right)
$$

is an isomorphism after tensoring with the rationals $\mathbb{Q}$.

Proof. Let $T \widehat{B U}_{k}$ denote the Thom space of the pullback of universal vector bundle $\gamma_{k}$ over $B O(k)$ to $\widehat{B U}\left(\left[\frac{k}{2}\right]\right)$ with $f_{k}=\left(j_{k-1}\right) \circ h_{\left[\frac{k}{2}\right]} \circ\left(\hat{\phi}_{N / t}\right)_{\left[\frac{k}{2}\right]}: \widehat{B U}\left(\left[\frac{k}{2}\right]\right) \rightarrow B O(k)$. Then by Theorem 3.17 and the generalized Pontrjagin-Thom Theorem (see $[\mathbf{1 7}$, p. 18]) one gets:

$$
\Omega_{n}^{U, N^{c, t}} \cong \lim _{k \rightarrow \infty} \pi_{n+k}\left(T \widehat{B U}_{k}, \infty\right)
$$

Since the Thom space $T \widehat{B U}_{k}$ is $(k-1)$-connected, therefore, by results of Serre (cf. [15, p. 207]), after tensoring with the rationals $\mathbb{Q}$, the Hurewicz homomorphism $\pi_{n+k}\left(T \widehat{B U}_{k}, \infty\right) \otimes \mathbb{Q} \rightarrow H_{n+k}\left(T \widehat{B U}_{k}, \infty, \mathbb{Z}\right) \otimes \mathbb{Q}$ is an isomorphism for $n+$ $k<2 k-1$, and hence for $k>n+1$. Because the map $f_{k}$ is induced by a complex vector bundle, the bundle $f_{k}^{*}\left(\gamma_{k}\right)$ is oriented, and so we have by Thom isomorphism that $H_{n+k}\left(T \widehat{B U}_{k}, \infty, \mathbb{Z}\right) \cong H_{n}\left(\widehat{B U}\left(\left[\frac{k}{2}\right]\right), \mathbb{Z}\right)$. Also, we get $H_{n}\left(\widehat{B U}\left(\left[\frac{k}{2}\right]\right), \mathbb{Q}\right) \cong$ $H_{n}\left(B U\left(\left[\frac{k}{2}\right] \times K(\mathbb{Z}, 2)\right), \mathbb{Q}\right)$ by applying the Leray spectral sequence to the fibration 


$$
\left(\hat{\phi}_{N / t}\right)_{\left[\frac{k}{2}\right]}: \widehat{B U}\left(\left[\frac{k}{2}\right]\right) \rightarrow B U\left(\left[\frac{k}{2}\right]\right) \times K(\mathbb{Z}, 2)
$$

with the fiber $K\left(\mathbb{Z}_{N / t}, 1\right)$ and $H_{*}\left(K\left(\mathbb{Z}_{N / t}, 1\right), \mathbb{Q}\right) \cong \mathbb{Q}$. Consequently, we have for $k>n+1$ that

$$
\begin{aligned}
\pi_{n+k}\left(T \widehat{B U}_{k}, \infty\right) \otimes \mathbb{Q} & \cong H_{n+k}\left(T \widehat{B U}_{k}, \infty, \mathbb{Z}\right) \otimes \mathbb{Q} \\
& \cong H_{n}\left(\widehat{B U}\left(\left[\frac{k}{2}\right]\right), \mathbb{Q}\right) \\
& \cong H_{n}\left(B U\left[\frac{k}{2}\right] \times K(\mathbb{Z}, 2), \mathbb{Q}\right) \\
& \cong \bigoplus_{i+j=n}\left(H_{i}\left(B U\left[\frac{k}{2}\right], \mathbb{Q}\right) \otimes H_{j}\left(\mathbb{C P}^{\infty}, \mathbb{Q}\right)\right) .
\end{aligned}
$$

The last isomorphism is by the Künneth theorem.

Since one has

$$
\Omega_{n}^{U}\left(\mathbb{C P}^{\infty}\right) \otimes \mathbb{Q} \cong \bigoplus_{i+j=n}\left(\Omega_{i}^{U} \otimes \mathbb{Q}\right) \otimes H_{j}\left(\mathbb{C P}^{\infty}, \mathbb{Q}\right),
$$

and also $\Omega_{i}^{U} \cong \lim _{k \rightarrow \infty} \pi_{i+k}\left(T B U_{k}, \infty\right)$, therefore, by using the previous arguments and applying the Serre theorem and the Thom isomorphism we get $\pi_{i+k}\left(T B U_{k}, \infty\right) \otimes \mathbb{Q} \cong$ $H_{i+k}\left(T B U_{k}, \infty, \mathbb{Z}\right) \otimes \mathbb{Q} \cong H_{i}\left(B U\left[\frac{k}{2}\right], \mathbb{Q}\right)$, for $k>i+1$. More precisely, now we have that the forgetful homomorphism is also a rational isomorphism.

\subsection{The group $\Omega_{n}^{U, N, t}(F) \otimes \mathbb{Q}$}

This subsection is devoted to finding a computable characterization of the rational bordism group $\Omega_{n}^{U, N, t}(F) \otimes \mathbb{Q}$ of $n$-dimensional closed complex $N$-Spin manifolds with free circle actions of type $t$ for $t \mid N$, which appears in the exact sequence (3.1).

Proposition 3.19. Let $M$ be a complex $N$-Spin manifold with a free circle action $\alpha$, and let $t \neq 0$ be such that $t \mid N$. Then the circle action $\alpha$ has type $t$ if and only if the orbit space $M / S^{1}$ can be given a complex $N$-Spin ${ }^{c, t}$ structure with the principal $U(1)$-bundle $\pi: M \rightarrow M / S^{1}$ and principal $U^{N, c, t}$-bundle given by the composition $E \rightarrow M \rightarrow M / S^{1}$, where $E \rightarrow M$ is the $\hat{U}(1)$-bundle over $M$ determined by the given complex $N$-Spin structure on $M$.

Proof. Let the complex $N$-Spin structure on $M$ be given by the following commutative diagram:

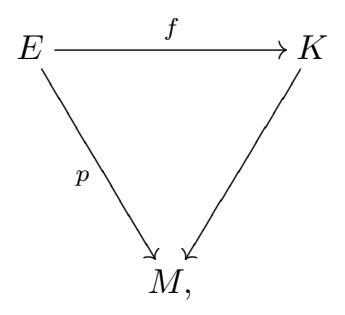

where $K$ is the $U(1)$-bundle associated to the stable tangent bundle representing the $U$-structure on $M, E$ is a $\hat{U}(1)$-bundle, and $f$ is the $N$-covering map.

Suppose that the circle action $\alpha$ has type $t$. Then the connected $N / t$-covering $\hat{S}^{1}$ of $S^{1}$ acts freely on $E$ and commutes with the right $\hat{U}(1)$-action on $E$, and is compatible with $f: E \rightarrow K$. Define an action $\hat{\alpha}$ of $\hat{U}(1) \times \hat{S}^{1}$ on $E$ by sending $(x,(g, \lambda))$ to $\lambda^{-1} x g$, 
where $x \in E, g \in \hat{U}(1)$, and $\lambda \in \hat{S}^{1}$. This is a free action of $\hat{U}(1) \times \hat{S}^{1}$ on $E$ and it induces a free action of $U^{N, c, t}$ on $E$. Moreover, the composition $\pi \circ p: E \rightarrow M \rightarrow$ $M / S^{1}$ is a $U^{N, c, t}$-bundle over $M / S^{1}$.

Now define a map $\mu: E \rightarrow K / S^{1} \times M$ as $\mu(x)=(\bar{\pi}(f(x)), p(x))$, where $\bar{\pi}: K \rightarrow$ $K / S^{1}$ is the orbit projection. The map $\mu$ is equivariant, and we have the following commutative diagram:

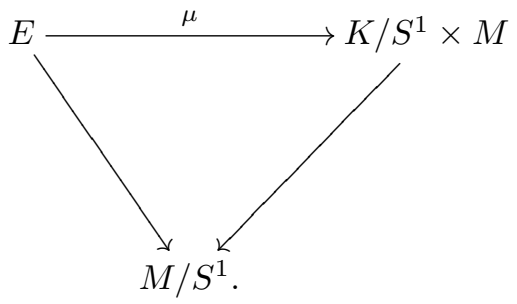

So we have a complex $N$-Spin ${ }^{c, t}$ structure on $K / S^{1}$ and hence on $M / S^{1}$.

Conversely assume that a complex $N$-Spin ${ }^{c, t}$ structure on $M / S^{1}$ induced by the complex $N$-Spin structure on $M$ is given by the following diagram:

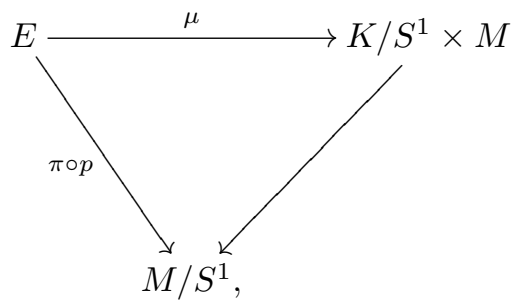

where $\mu$ sends an element $x$ of $E$ to the element $(\bar{\pi}(f(x)), p(x))$ of $K / S^{1} \times M$. Let us consider the following commutative pullback diagram:

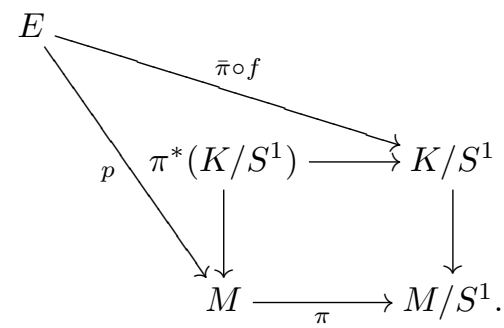

Define a map $\nu: E \rightarrow \pi^{*}\left(K / S^{1}\right)$ by $\nu(x)=(\bar{\pi}(f(x)), p(x))$, for $x \in E$. Because the map $\mu: E \rightarrow K / S^{1} \times M$ is an $N$-covering, therefore, the diagram

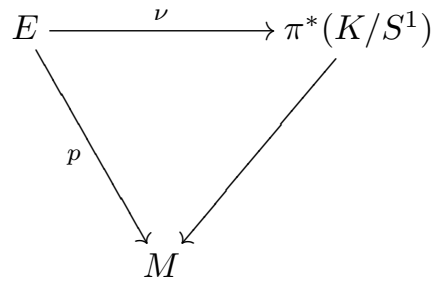

defines a complex $N$-Spin structure on $M$ which, under the identification $K \cong$ 
$\pi^{*}\left(K / S^{1}\right)$, coincides with the given complex $N$-Spin structure on $M$. We need to show that the circle action $\alpha$ is of type $t$. Let $x \in E$ and the map

$$
\alpha_{\nu(x)}: \mathbb{R} \rightarrow \pi^{*}\left(K / S^{1}\right)
$$

induced by $\alpha$ be given by

$$
\alpha_{\nu(x)}(s)=\left(\bar{\pi}(f(x)), \alpha\left(e^{2 \pi i s}, p(x)\right)\right) .
$$

Also, define

$$
\hat{\alpha}_{\nu(x)}: \mathbb{R} \rightarrow E
$$

by

$$
\hat{\alpha}_{\nu(x)}(s)=x \cdot\left[1, e^{\frac{2 \pi t i s}{N}}\right],
$$

where $\left[1, e^{\frac{2 \pi t i s}{N}}\right] \in U^{N, c, t}$. We see that $\hat{\alpha}_{\nu(x)}(0)=x$, and $\nu\left(\hat{\alpha}_{\nu(x)}(s)\right)=\nu\left(x \cdot\left[1, e^{\frac{2 \pi t i s}{N}}\right]\right)$ $=\mu\left(x \cdot\left[1, e^{\frac{2 \pi t i s}{N}}\right]\right)=\left(\bar{\pi}(f(x)), \alpha\left(e^{2 \pi i s}, p(x)\right)\right)=\alpha_{\nu(x)}(s)$. Therefore, $\hat{\alpha}_{\nu(x)}$ is a lifting of $\alpha_{\nu(x)}$, and $\hat{\alpha}_{\nu(x)}(1)=x \cdot\left[1, e^{\frac{2 \pi i t}{N}}\right]$. Thus the circle action $\alpha$ is of type $t$.

The above proposition can be translated in terms of bordism language as follows.

Lemma 3.20. For $t \neq 0$ and $t \mid N$, we have an isomorphism

$$
\Omega_{n}^{U, N, t}(F) \rightarrow \Omega_{n-1}^{N^{c, t}}
$$

given by

$$
[M] \mapsto\left[M / S^{1}\right] .
$$

A direct consequence of Theorem 3.18 and Lemma 3.20 is:

Theorem 3.21. If $t \neq 0$ and $t \mid N$, then the homomorphism

$$
\Omega_{n}^{U, N, t}(F) \rightarrow \Omega_{n-1}^{U}\left(\mathbb{C P}^{\infty}\right)
$$

given by

$$
[M] \mapsto\left[M / S^{1}, f\right]
$$

is an isomorphism after tensoring with the rationals $\mathbb{Q}$, where $f$ is the classifying map of $S^{1}$-bundle $M \rightarrow M / S^{1}$.

\section{Applications}

In this section we will discuss a few applications of the theory developed in the previous section to explore the ideal $I_{*}^{N, t}$ in the rational bordism ring $\Omega_{*}^{U, N} \otimes \mathbb{Q}$ generated by bordism classes of complex $N$-Spin manifolds admitting an effective circle action of type $t$. We will find a condition to determine the ideal $I_{*}^{N, t}$ and make computations to show that the condition holds true for several values of $N$ and $t$, and thereby verify the conjectural equation (1.1) for those values of $N$ and $t$. More precisely, we will see that the conjectural equation holds true for all values of $t$ with $N \leqslant 9$, except for case $(N, t)=(6,3)$ which remains undetermined. 


\subsection{Exact sequence}

In this subsection we will set up a general framework for computations to explore the ideal $I_{*}^{N, t}$ by exploiting characterizations, obtained in the previous section, of the bordism groups in the exact sequence (3.1).

Composing the isomorphisms obtained from Theorem 3.13 and Theorem 3.21 suitably with the maps $i_{N, t}, j_{N, t}$, and $\partial_{N, t}$ of the exact sequence (3.1) we get maps $\bar{i}_{N, t}$, $\bar{j}_{N, t}$, and $\bar{\partial}_{N, t}$, respectively, which we will denote with an abuse of notation simply by $i_{N, t}, j_{N, t}$ and $\partial_{N, t}$, respectively. Thus we have:

Lemma 4.1. The sequence

$$
\begin{aligned}
& \cdots \quad \Omega_{n-1}^{U}\left(\mathbb{C P}^{\infty}\right) \otimes \mathbb{Q} \quad \stackrel{i_{N, t}}{\longrightarrow} \Omega_{n}^{U, N, t}(S F) \otimes \mathbb{Q} \\
& \stackrel{j_{N, t}}{\longrightarrow} \underset{\substack{k+2 p+2 q=n \\
p-q \equiv t(\bmod N)}}{\longrightarrow} \Omega_{k}^{U}(B U(p) \times B U(q)) \otimes \mathbb{Q} \stackrel{\partial_{N, t}}{\longrightarrow} \Omega_{n-2}^{U}\left(\mathbb{C P}^{\infty}\right) \otimes \mathbb{Q} \longrightarrow \cdots
\end{aligned}
$$

is exact.

Now we recall from $[\mathbf{9}]$, a few details about the complex twisted projective bundles needed for the subsequent development of this article. Let $E$ and $G$ be two complex vector bundles of rank $p$ and $q$ over a $U$-manifold $B$, respectively. The $U$-structure on $B$ and the complex structure on $E \oplus G$ provide a $U$-structure $\sigma^{*}(E \oplus G) \oplus \sigma^{*} \tau(B)$ on $E \oplus G \stackrel{\sigma}{\longrightarrow} B$ and - after a choice of Hermitian metric — on the disk bundle $D(E \oplus G)$, and the sphere bundle $S(E \oplus G)=\partial D(E \oplus G)$. Consider on $E \oplus G$ the $S^{1}$-action $\alpha: S^{1} \times E \oplus G \rightarrow E \oplus G$ given by $\alpha\left(\lambda,\left(p, e_{p}, g_{p}\right)\right)=\left(p, \lambda e_{p}, \lambda^{-1} g_{p}\right)$. This $S^{1}$-action restricted to $S(E \oplus G)$ is free and respects the $U$-structure. The quotient $S(E \oplus G) / \alpha$ as a differentiable manifold is the complex projective bundle $\mathbb{C P}(E \oplus$ $\bar{G})$, where $\bar{G}$ is the complex bundle conjugate to $G$. The twisted projective bundle $\widetilde{\mathbb{C P}}(E \oplus G)$ of two complex vector bundles $E$ and $G$ over a $U$-manifold $B$ is defined as the bundle $\mathbb{C P}(E \oplus \bar{G})$ with the stably almost complex structure $\tau(\widetilde{\mathbb{C P}}(E \oplus G)):=$ $\left(S^{*} \otimes \pi^{*} E \oplus \overline{S^{*}} \otimes \pi^{*} G \oplus \pi^{*} \tau(B)\right)$, where $S^{*}$ is the dual bundle of the tautological line bundle $S$, and $\pi: \mathbb{C P}(E \oplus \bar{G}) \rightarrow B$ is the projection map. The orientation of $\widetilde{\mathbb{C P}}(E \oplus G)$ induced by this "twisted" stably almost complex structure is $(-1)^{q}$ times the usual orientation of $\mathbb{C P}(E \oplus \bar{G})$. The cohomology of $\widetilde{\mathbb{C P}}(E \oplus G)$ is the cohomology of $\mathbb{C P}(E \oplus \bar{G})$ :

$$
H^{*}(\widetilde{\mathbb{C P}}(E \oplus G)) \simeq H^{*}(B)[t] /\left\langle t^{p+q}+c_{1}(E \oplus \bar{G}) t^{p+q-1}+\cdots+c_{p+q}(E \oplus \bar{G})\right\rangle,
$$

where $c_{i}$ stands for the $i$-th Chern class, and $t=c_{1}\left(S^{*}\right)$ denotes the first Chern class of the dual bundle of the tautological line bundle $S$. The $S^{1}$-bundle $S(E \oplus G)$ over $\widetilde{\mathbb{C P}}(E \oplus G)$ under the given action $\alpha$ has the first Chern class $-t$. If $h$ is the $S^{1}$-principal bundle classifying map, then $[\widetilde{\mathbb{C P}}(E \oplus G), h]$ defines an element in $\Omega_{*}^{U}\left(\mathbb{C P} \mathbb{P}^{\infty}\right)$. The bordism group $\Omega_{*}^{U}\left(\mathbb{C P}^{\infty}\right)$ as a $\Omega_{*}^{U}$-module is isomorphic to the bordism group $\Omega_{*+1}^{U}(F)$ of $U$-manifolds with free circle actions. The stably almost complex structure on $\widetilde{\mathbb{C P}}(E \oplus G)$ was chosen such that $[\widetilde{\mathbb{C P}}(E \oplus G), h]$ under the above isomorphism of $S^{1}$-manifolds is equivalent to $[S(E \oplus G), \alpha]$.

Let $v_{i}^{+}$and $v_{i}^{-}$have the same meanings as in remarks preceding Proposition 3.2. Define $k_{U}: \Omega_{n}^{U}(S F, F) \rightarrow \Omega_{n}^{U}(S F)$ by $[M] \mapsto \sum_{i}\left[\widetilde{\mathbb{C P}}\left(\left(v_{i}^{+} \oplus \epsilon_{\mathbb{C}}^{1}\right) \oplus v_{i}^{-}\right)\right]$, where $\epsilon_{\mathbb{C}}^{1}$ is 
the trivial complex line bundle. If $j_{U}$ is the inclusion map $\Omega_{n}^{U}(S F) \rightarrow \Omega_{n}^{U}(S F, F)$, then $k_{U} \circ j_{U}=$ id (see, for instance, [7]). Now, in particular, if $[M] \in \Omega_{n}^{U}(S F)$, then $[M]=k_{U} \circ j_{U}[M]=k_{U}\left(j_{U}[M]\right)=k_{U}[M]$, therefore, the equation

$$
[M]=\sum_{i}\left[\widetilde{\mathbb{C P}}\left(\left(v_{i}^{+} \oplus \epsilon_{\mathbb{C}}^{1}\right) \oplus v_{i}^{-}\right)\right]
$$

holds in $\Omega_{n}^{U}(S F)$.

Let

$$
\varepsilon_{N, t}: \quad \bigoplus_{\substack{k+2 p+2 q=n \\ p-q \equiv t(\bmod N)}} \Omega_{k}^{U}(B U(p) \times B U(q)) \otimes \mathbb{Q} \rightarrow \Omega_{n}^{U} \otimes \mathbb{Q}
$$

be the linear map defined by sending $[X, E \oplus G]$ to $\left[\widetilde{\mathbb{C P}}\left(\left(E \oplus \epsilon_{\mathbb{C}}^{1}\right) \oplus G\right)\right]$.

Lemma 4.2. If there exist bordism classes $\left[U_{1}\right],\left[U_{2}\right], \ldots,\left[U_{m}\right] \in \operatorname{Im}\left(\left.\varepsilon_{N, t}\right|_{\operatorname{ker}\left(\partial_{N, t}\right)}\right)$ such that

$$
P^{N, t} \subset\left\langle\varphi_{\mathrm{ell}}\left[U_{1}\right], \varphi_{\mathrm{ell}}\left[U_{2}\right], \ldots, \varphi_{\mathrm{ell}}\left[U_{m}\right]\right\rangle
$$

then we have

$$
I_{*}^{N, t}=\bigcap_{n \mid N, n \nmid t} \operatorname{ker} \varphi_{n} .
$$

Proof. Let $\left[U_{i}^{\prime}\right] \in \operatorname{ker}\left(\partial_{N, t}\right)$ be such that $\varepsilon_{N, t}\left(\left[U_{i}^{\prime}\right]\right)=\left[U_{i}\right]$. By the exactness of sequence in Lemma 4.1 , there exists a $\left[V_{i}\right] \in \Omega_{n}^{U, N, t}(S F) \otimes \mathbb{Q}$ such that $j_{N, t}\left(\left[V_{i}\right]\right)=$ $\left[U_{i}^{\prime}\right]$. By equation (4.2), the Chern numbers of $\left[V_{i}\right]$ and $\left[U_{i}\right]$ are the same, and hence $\varphi_{\text {ell }}\left[V_{i}\right]=\varphi_{\text {ell }}\left[U_{i}\right]$. So there exist bordism classes $\left[V_{1}\right],\left[V_{2}\right], \ldots,\left[V_{m}\right] \in I_{*}^{N, t}$ such that $P^{N, t} \subset\left\langle\varphi_{\mathrm{ell}}\left[V_{1}\right], \varphi_{\mathrm{ell}}\left[V_{2}\right], \ldots, \varphi_{\mathrm{ell}}\left[V_{m}\right]\right\rangle$. The result follows by Proposition 2.4.

\subsection{The ideals $I_{*}^{N, t}$}

In this subsection we study the ideal $I_{*}^{N, t}$ in the ring $\Omega^{N, t} \otimes \mathbb{Q}$ generated by bordism classes of complex $N$-Spin manifolds with an effective circle action of type $t$. More precisely, we verify the conjectural equation (1.1) for several values of $N$ and $t$ by constructing bordism classes of manifolds satisfying the sufficient condition of Lemma 4.2. We begin the case $N=4$ and $t=2$ with an explicit proof including details of its various important steps to present the general technique which will work for higher values of $N$ and $t$, as well.

Theorem 4.3. Let $\varphi_{4}$ denote the elliptic genus of level 4 . Then we have

$$
I_{*}^{4,2}=\operatorname{ker}\left(\varphi_{4}\right) .
$$

Proof. Consider the linear map

$$
\partial_{4,2}: \bigoplus_{\substack{k+p+q=4 \\ p-q \equiv 2(\bmod 4)}} \Omega_{2 k}^{U}(B U(p) \times B U(q)) \otimes \mathbb{Q} \rightarrow \Omega_{6}^{U}\left(\mathbb{C P}^{\infty}\right) \otimes \mathbb{Q} .
$$

We intend to compute its kernel. Let $E_{i}$ and $G_{i}$ be complex vector bundles over $U$ manifold $B_{i}, 1 \leqslant i \leqslant 4$, where $B_{1}$ and $B_{2}$ are zero dimensional $U$-manifolds with the number of elements $r$ and $s$ respectively, and $B_{3}$ and $B_{4}$ are 2-dimensional manifolds. If the bundles $E_{1}, E_{2}, E_{3}, E_{4}$ are of $3,1,2,0$ complex ranks, and $G_{1}, G_{2}, G_{3}, G_{4}$ 
are of 1, 3, 0, 2 complex ranks, then $\left[E_{1} \oplus G_{1}, B_{1}\right]+\left[E_{2} \oplus G_{2}, B_{2}\right]+\left[E_{3} \oplus G_{3}, B_{3}\right]+$ $\left[E_{4} \oplus G_{4}, B_{4}\right]$ is a well-defined element of $\bigoplus_{k+p+q=4} \Omega_{2 k}^{U}(B U(p) \times B U(q))$ and its rational image under $\partial_{4,2}$ is given by $p-q \equiv 2(\bmod 4)$

$$
\left[\widetilde{\mathbb{C P}}\left(E_{1} \oplus G_{1}\right), h_{1}\right]+\left[\widetilde{\mathbb{C P}}\left(E_{2} \oplus G_{2}\right), h_{2}\right]+\left[\widetilde{\mathbb{C P}}\left(E_{3} \oplus G_{3}\right), h_{3}\right]+\left[\widetilde{\mathbb{C P}}\left(E_{4} \oplus G_{4}\right), h_{4}\right] .
$$

Consider the total Chern classes $c\left(B_{3}\right)=1+a_{1}\left(B_{3}\right)+a_{2}\left(B_{3}\right), c\left(B_{4}\right)=1+b_{1}\left(B_{4}\right)+$ $b_{2}\left(B_{4}\right), \quad c\left(E_{3}\right)=1+e_{1}\left(E_{3}\right)+e_{2}\left(E_{3}\right), \quad c\left(G_{4}\right)=1+g_{1}\left(G_{4}\right)+g_{2}\left(G_{4}\right) . \quad$ An element $[M, h] \otimes 1$ of $\Omega_{6}^{U}\left(\mathbb{C P}^{\infty}\right) \otimes \mathbb{Q}$ is zero if all of its generalized Chern numbers $c_{3}, c_{2} c_{1}$, $c_{1}^{3}, c_{2} d, c_{1}^{2} d, c_{1} d^{2}$, and $d^{3}$ are zero, where $d=h^{*}(g)$ in $H^{2}(M, \mathbb{Z})$ is the pullback of the generator of $H^{*}\left(\mathbb{C P}^{\infty}, \mathbb{Z}\right) \cong \mathbb{Z}[g]$. By using the equation (4.1), the generalized Chern numbers of the expression in (4.3) are given by

$$
\begin{aligned}
c_{3} & =2 r-2 s+2 a_{2}-2 b_{2}, \\
c_{2} c_{1} & =2 a_{1}^{2}+2 a_{2}-2 b_{1}^{2}-2 b_{2}, \\
c_{1}^{3} & =-8 r+8 s+6 a_{1}^{2}+2 e_{1}^{2}-8 e_{2}-6 b_{1}^{2}-2 g_{1}^{2}+8 g_{2}, \\
c_{2} d & =-a_{2}+a_{1} e_{1}-b_{2}+b_{1} g_{1}, \\
c_{1}^{2} d & =4 r+4 s-a_{1}^{2}-e_{1}^{2}+4 e_{2}+2 a_{1} e_{1}-b_{1}^{2}-g_{1}^{2}+4 g_{2}+2 b_{1} g_{1}, \\
c_{1} d^{2} & =-2 r+2 s+e_{1}^{2}-2 e_{2}-a_{1} e_{1}-g_{1}^{2}+2 g_{2}+b_{1} g_{1}, \\
d^{3} & =r+s-e_{1}^{2}+e_{2}-g_{1}^{2}+g_{2} .
\end{aligned}
$$

Each solution of the system, obtained from the above system by putting left sides of equations equal to zero, determines an element of the kernel of $\partial_{4,2}$. The solution set of the system is spanned by $(-5,-3,-14,2,-10,-2,0,-12,0,0,0,2),(-1,-1,0,0,0,1$, $0,0,0,0,1,0),(1,1,3,0,1,0,0,3,0,1,0,0),(7,3,20,-2,12,2,0,16,2,0,0,0), \quad(-1,0$, $-1,1,-1,0,1,0,0,0,0,0)$ with respect to the coordinates $\left(r, s, a_{1}^{2}, a_{2}, e_{1}^{2}, e_{2}, a_{1} e_{1}, b_{1}^{2}\right.$, $\left.b_{2}, g_{1}^{2}, g_{2}, b_{1} g_{1}\right)$.

Consider the linear map

$$
\varepsilon_{4,2}: \bigoplus_{\substack{k+p+q=4 \\ p-q \equiv 2(\bmod 4)}} \Omega_{2 k}^{U}(B U(p) \times B U(q)) \otimes \mathbb{Q} \rightarrow \Omega_{8}^{U} \otimes \mathbb{Q} .
$$

We intend to compute $\operatorname{Im}\left(\left.\varepsilon_{4,2}\right|_{\operatorname{ker}\left(\partial_{4,2}\right)}\right)$. Let $E_{i}, G_{i}, 1 \leqslant i \leqslant 4$ be as above, then the Chern numbers of the bordism class $\left[\widetilde{\mathbb{C P}}\left(\left(E_{1} \oplus \epsilon_{\mathbb{C}}^{1}\right) \oplus G_{1}\right)\right]+\left[\widetilde{\mathbb{C P}}\left(\left(E_{2} \oplus \epsilon_{\mathbb{C}}^{1}\right) \oplus G_{2}\right)\right]+$ $\left[\widetilde{\mathbb{C P}}\left(\left(E_{3} \oplus \epsilon_{\mathbb{C}}^{1}\right) \oplus G_{3}\right)\right]+\left[\widetilde{\mathbb{C P}}\left(\left(E_{4} \oplus \epsilon_{\mathbb{C}}^{1}\right) \oplus G_{4}\right)\right]$ are given by

$$
\begin{aligned}
c_{4} & =3 r-s+3 a_{2}-b_{2}, \\
c_{3} c_{1} & =6 r+2 s+3 a_{1}^{2}+9 a_{2}-b_{1}^{2}+b_{2}, \\
c_{2}^{2} & =-4 r-4 s+9 a_{1}^{2}+6 a_{2}+e_{1}^{2}-3 e_{2}+b_{1}^{2}-2 b_{2}+g_{1}^{2}-3 g_{2}, \\
c_{2} c_{1}^{2} & =-18 r+2 s+21 a_{1}^{2}+9 a_{2}+6 e_{1}^{2}-18 e_{2}+b_{1}^{2}+b_{2}+2 b_{1} g_{1}, \\
c_{1}^{4} & =-81 r-s+54 a_{1}^{2}+27 e_{1}^{2}-81 e_{2}+6 b_{1}^{2}+3 g_{1}^{2}-g_{2}+8 b_{1} g_{1} .
\end{aligned}
$$

One obtains that the image $\operatorname{Im}\left(\varepsilon_{4,2} \mid \operatorname{ker}\left(\partial_{4,2}\right)\right)$ is spanned by $\left[U_{1}\right]=(1,4,-1,0,0)$ and $\left[U_{2}\right]=(0,3,13,28,64)$, where the coordinates are the Chern numbers $\left(c_{4}, c_{3} c_{1}, c_{2}^{2}\right.$, $\left.c_{2} c_{1}^{2}, c_{1}^{4}\right)$. The polynomials of degrees three and four associated to the multiplicative 
sequence of the genus $\varphi_{\text {ell }}$ are given by:

$$
\begin{aligned}
K_{3}= & \frac{1}{2^{5} \cdot 3}\left(48 C c_{3}+(2 A B-48 C) c_{2} c_{1}+\left(2 A^{3}-A B+16 C\right) c_{1}^{3}\right) \quad \text { and } \\
K_{4}= & \frac{1}{2^{9} \cdot 3^{2} \cdot 5}\left(\left(-8 B^{2}+4608 D\right) c_{4}+\left(5760 A C+8 B^{2}-4608 D\right) c_{3} c_{1}\right. \\
& +\left(24 B^{2}-2304 D\right) c_{2}^{2}+\left(120 A^{2} B-5760 A C-28 B^{2}+4608 D\right) c_{1}^{2} c_{2} \\
& \left.+\left(60 A^{4}-60 A^{2} B+1920 A C+7 B^{2}-1152 D\right) c_{1}^{4}\right)
\end{aligned}
$$

(see, for instance, $[\mathbf{9}])$. It follows that $\varphi_{\text {ell }}\left(\left[\mathbb{C P}^{3}\right]\right)=(2 C) / 3-(A B) / 6+\left(4 A^{3}\right) / 3$ and $\varphi_{\mathrm{ell}}\left(\left[U_{1}\right]\right)=A C-D / 2$. Since $P^{4,2}=\left\langle 2 A C-D, 8 A^{3}-A B+4 C\right\rangle$ by remarks preceding Definition 2.2, we see that $P^{4,2} \subset\left\langle\varphi_{\text {ell }}\left(\left[U_{1}\right]\right), \varphi_{\text {ell }}\left(\left[\mathbb{C P}^{3}\right]\right)\right\rangle$. Lemma 4.2 finishes the proof.

We will see that the conjectural equation (1.1) also holds true for a few higher values of $N$. We can give proofs for those cases of higher values of $N$ along lines of the above proof. However, to simplify their presentation we will use the idea of Hilbert-Poincaré series of a graded ideal of $\mathbb{C}[A, B, C, D]$. Let $J=\bigoplus_{n \in \mathbb{N}} J_{n}$ be a graded ideal in $\mathbb{C}[A, B, C, D]$, where each $J_{n}$ containing elements of degree $n$ has the finite dimension, say, $a_{n}$. The Hilbert-Poincare series of $J$ in indeterminate $s$ is given by $\sum_{n \in \mathbb{N}} a_{n} s^{n}$. We make an observation here which will be useful in formulating a simple proof of the conjecture for a few higher values of $N$ : Let $J^{\prime} \subset J$ be an inclusion of graded ideals in $\mathbb{C}[A, B, C, D]$, and let $J$ be generated by a finite number of elements with the highest degree of its generators equal to $n_{0}$. If $a_{n}^{\prime}=a_{n}$ for all $n \leqslant n_{0}$, then we have $J^{\prime}=J$.

From now on we will be more specific about notations of maps $\varepsilon_{N, t}$ and $\partial_{N, t}$, and will denote them by $\varepsilon_{N, t}^{(n)}$ and $\partial_{N, t}^{(n)}$, respectively, when their domains of definition have degree $n$. We will denote the image $\varphi_{\text {ell }}\left(\operatorname{Im}\left(\left.\varepsilon_{N, t}^{(n)}\right|_{\operatorname{ker}\left(\partial_{N, t}^{(n)}\right)}\right)\right)$ of the universal elliptic genus by $P_{n}^{N^{N, t}}$. Let ${P^{\prime N, t}}^{N,} \bigoplus_{n \in \mathbb{N}}{P_{n}^{\prime N, t}}^{N}$ and $P^{N, t}=\bigoplus_{n \in \mathbb{N}} P_{n}^{N, t}$ be graded ideals of $\mathbb{C}[A, B, C, D]$ with dimensions of $P_{n}^{\prime^{N, t}}$ and $P_{n}^{N, t}$ denoted by $d_{n}^{\prime}$ and $d_{n}$, respectively. We remark that $P^{\prime^{N, t}} \subset P^{N, t}$. In fact, a generic element of $P^{\prime^{N, t}}$ has the form $\varphi_{\text {ell }}[U]$, where $[U] \in \operatorname{Im}\left(\left.\varepsilon_{N, t}\right|_{\operatorname{ker}\left(\partial_{N, t}\right)}\right)$. As in the context of the proof of Lemma 4.2, there exists $[V] \in I_{*}^{N, t}$ such that $\varphi_{\text {ell }}[U]=\varphi_{\text {ell }}[V]$. Since $I_{*}^{N, t} \subset \bigcap_{n \mid N, n \nmid t} \operatorname{ker} \varphi_{n}$ (see Section 2), we have $[V] \in \bigcap_{n \mid N, n \nmid t} \operatorname{ker} \varphi_{n}$, and so it follows by Proposition 2.3 that $[V] \in \operatorname{ker} \psi_{N, t}$. By equation (2.4), we get $[V] \in \operatorname{ker}\left(\pi_{N, t} \circ \varphi_{\text {ell }}\right)$. Hence $\pi_{N, t}\left(\varphi_{\text {ell }}[V]\right)$ is the zero element and so $\varphi_{\text {ell }}[V] \in \operatorname{ker} \pi_{N, t}=P^{N, t}$. Hence $\varphi_{\text {ell }}[U] \in P^{N, t}$, and as it was a generic element of $I_{N, t}^{\prime}$, we have the inclusion $P^{\prime^{N, t}} \subset P^{N, t}$. This remark and the above observation will be used in proving the following result which will give a general framework to present a simplified proof of the conjecture for several values of $N$ and $t$.

Proposition 4.4. Let $n_{0}$ be the highest degree of generators of $P^{N, t}$. If $d_{n}=d_{n}^{\prime}$ for $n \leqslant n_{0}$. Then

$$
I_{*}^{N, t}=\bigcap_{n \mid N, n \nmid t} \operatorname{ker} \varphi_{n} .
$$


Proof. Since $P^{\prime^{N, t}} \subset P^{N, t}$, therefore, hypothesis of the proposition together with the above observation imply that $P^{\prime^{N, t}}=P^{N, t}$. It follows from this equation and the definition of $P^{\prime^{N, t}}$ that the sufficient condition of Lemma 4.2 pertaining to the existence of bordism classes and inclusion is satisfied with equality. This completes the proof.

Theorem 4.5. The dimensions $d_{n}$ and $d_{n}^{\prime}$ of $P_{n}^{N, t}$ and $P_{n}^{N^{N, t}}$, respectively, up to the degree $n=12$, for several values of $N$ and $t$ are given in Table $1 .^{2}$

Table 1: Dimensions $d_{n}$ and $d_{n}^{\prime}$ of $P_{n}^{N, t}$ and $P_{n}^{\prime N, t}$, respectively

\begin{tabular}{|c||c|c|c|c|c|c|c|c|c|c|c|c|}
\hline$(N, t) / n$ & 1 & 2 & 3 & 4 & 5 & 6 & 7 & 8 & 9 & 10 & 11 & 12 \\
\hline \hline$(4,2)$ & & & 1 & 2 & 3 & 5 & 7 & 10 & 13 & 17 & 21 & 27 \\
\hline$(6,2)$ & & & & & 1 & 1 & 3 & 5 & 7 & 10 & 14 & 18 \\
\hline$(6,3)$ & & & & & 1 & 2 & 4 & $6(\mathbf{5})$ & 9 & $12 \mathbf{( 1 1 )}$ & 16 & $21(\mathbf{1 9 )}$ \\
\hline$(8,2)$ & & & & & & & 1 & 1 & 3 & 4 & 7 & 10 \\
\hline$(8,4)$ & & & & & & & 1 & 2 & 4 & 6 & 9 & 13 \\
\hline$(9,3)$ & & & & & & & & 1 & 1 & 3 & 4 & 8 \\
\hline$(10,2)$ & & & & & & & & & 1 & 1 & 3 & 4 \\
\hline$(10,5)$ & & & & & & & & & 1 & 2 & 4 & 6 \\
\hline
\end{tabular}

The dimensions $d_{n}^{\prime}$ are made bold and given in Table 1 only when they differ from the dimensions $d_{n}$. The entries in the table containing zeros are kept empty. Now we will use the highest degree of generators of $P^{N, t}$ to obtain the following result. In most cases we can verify that $P^{N, t}$ is generated by three elements whose degrees can be determined. But we will not need to use this fact in the proof, however, we will discuss it later for extra information.

Theorem 4.6. For $(N, t)=(6,2),(8,2),(8,4)$, and $(9,3)$, we have

$$
I_{*}^{N, t}=\bigcap_{n \mid N, n \nmid t} \operatorname{ker} \varphi_{n} .
$$

Proof. The highest degrees of generators of $P^{6,2}, P^{8,2}, P^{8,4}$, and $P^{9,3}$ are $8,12,9$, and 12, respectively. ${ }^{3}$ The result follows from Proposition 4.4 and Theorem 4.5.

Our technique does not work for higher values of $N$ because computer runs out of memory while running Mathematica program to compute dimensions $d_{n}^{\prime}$ of $P_{n}^{\prime^{N, t}}$ for higher degrees $n$. For instance, the highest degree of generators of $P^{10,2}$ is 16 and this degree is too big for the Mathematica program to compute $d_{16}^{\prime}$ for $(N, t)=(10,2)$. The technique developed in our work does not generate enough bordism classes in case of $(N, t)=(6,3)$ to verify the sufficient condition of Lemma 4.2. It is our conjecture that the technique gives enough bordism classes except when $(N, t)=(4 k+2, k+1)$ for $k \geqslant 1$, however, Mathematica program does not work in computations of dimensions $d_{n}^{\prime}$ of $P_{n}^{\prime^{N, t}}$ for some degrees $n$ needed for higher values of $N$. Our technique works if

${ }^{2}$ The computations were accomplished with the help of computer programs in Mathematica and MAGMA.

${ }^{3}$ The computations were accomplished with the help of computer programs in Mathematica and MAGMA. 
$t=1$ or $t$ does not divide $N$, and Mathematica computations can be made to verify the conjecture for values of $N$ up to 9, as well. However, we combine Theorem 4.3, Theorem 4.6, and a result of [9] concerning a verification of the conjectural equation (1.1) in the case $t=1$ to state the following result.

Theorem 4.7. Let $t$ be a divisor of $N$ such that $t \neq N$ and $2 \leqslant N \leqslant 9$, except for $(N, t)=(6,3)$. We have

$$
I_{*}^{N, t}=\bigcap_{n \mid N, n \nmid t} \operatorname{ker} \varphi_{n} .
$$

Let $T_{N, t}(s)$ denote the product of the polynomial $(1-s)\left(1-s^{2}\right)\left(1-s^{3}\right)\left(1-s^{4}\right)$ and the Hilbert-Poincare series of the quotient algebra $\mathbb{C}[A, B, C, D] / P^{N, t}$ in the indeterminate $s$. We conjecture that in most cases the ideal $P^{N, t}$ is generated at most by three elements. More precisely, we have:

Conjecture 4.8. Let $t$ be a divisor of $N \geqslant 2$ and $(N, t) \neq(4 k+2, k+1), k \geqslant 1$. Then

(i) $T_{N, t}(s)$ is a polynomial of degree $2 N-t+1$ given by the equation

$$
T_{N, t}(s)=1-s^{N-1}-s^{N+1}-s^{2 N-2 t}+s^{2 N-t-1}+s^{2 N-t+1},
$$

(ii) a minimal generating set of $P^{N, t}$ has a generator in degree $n$ precisely when the polynomial $T_{N, t}(s)$ contains a term $s^{n}$ with the coefficient -1 .

Observe that a cancellation occurs in equation (4.4) when $t=1$ or $(N, t)=(4,2)$, and in these cases $P^{N, t}$ can be generated by two elements. We have verified the above conjecture up to $N=18$ with the help of computations. ${ }^{3}$

Notice that our technique seems to break down in verifying the conjectural equation (1.1) when $(N, t)=(4 k+2, k+1), k \geqslant 1$ (cf. $(N, t)=(6,3)$ in Table 1$)$. These values of $N$ and $t$ are consistent with two facts, namely, the values of $N$ and $t$ for which $T_{N, t}(s)$ does not satisfy the equation (4.4) and $d_{n}^{\prime}$ is smaller than $d_{n}$. For $(N, t)=$ $(4 k+2, k+1), 1 \leqslant k \leqslant 4$, we have

$$
\begin{aligned}
T_{6,3}(s) & =1-s^{5}-s^{6}-s^{7}+s^{9}+s^{10}+s^{11}-s^{12} \\
T_{10,5}(s) & =1-s^{9}-s^{10}-s^{11}+s^{14}+s^{17}+s^{19}-s^{20}, \\
T_{14,7}(s) & =1-s^{13}-s^{14}-s^{15}+s^{20}+s^{22}-s^{24}+s^{25}+s^{27}-s^{28} \\
T_{18,9}(s) & =1-s^{17}-s^{18}-s^{19}-s^{21}-s^{23}+2 s^{25}+2 s^{26}+2 s^{27}-s^{28}-s^{30} .
\end{aligned}
$$

Let $b_{n}$ and $b_{n}^{\prime}$ denote the coefficients of Hilbert-Poincaré series of ideals $\bigcap_{n \mid N, n \nmid t} \operatorname{ker} \varphi_{n}$ and $\operatorname{Im}\left(\left.\varepsilon_{N, t}\right|_{\operatorname{ker}\left(\partial_{N, t}\right)}\right)=\bigoplus_{n \in \mathbb{N}} \operatorname{Im}\left(\left.\varepsilon_{N, t}^{(n)}\right|_{\operatorname{ker}\left(\partial_{N, t}^{(n)}\right)}\right)$ in $\Omega_{*}^{U, N} \otimes \mathbb{Q}$, respectively.

Theorem 4.9. The coefficients $b_{n}$ and $b_{n}^{\prime}$ up to degree 12 for several values of $N$ and $t$ are given in Table 2. ${ }^{4}$

The coefficients $b_{n}^{\prime}$ are given and made bold in the table only when they differ from the coefficients $b_{n}$. The entries in the table containing zeros are kept empty. Table 2 suggests the following conjecture:

${ }^{4}$ The computations were accomplished with the help of computer programs in Mathematica and MAGMA. 
Table 2: Values of $b_{n}$ and $b_{n}^{\prime}$

\begin{tabular}{|c|c|c|c|c|c|c|c|c|c|c|c|c|}
\hline$(N, t) / n$ & $|1|$ & 2 & \begin{tabular}{|l|l}
3 \\
\end{tabular} & 4 & 5 & 6 & 7 & 8 & 9 & 10 & 11 & 12 \\
\hline$(2,1)$ & 1 & 1 & \begin{tabular}{|l|l}
3 \\
\end{tabular} & 3 & 7 & 9 & 15 & 19 & 30 & 39 & 56 & 73 \\
\hline$(3,1)$ & & 1 & \begin{tabular}{|l|l|} 
& \\
\end{tabular} & 3 & 5 & 8 & 12 & 19 & 26 & 38 & 52 & 72 \\
\hline$(4,1)$ & & & 1 & 1 & 4 & 6 & 11 & 15 & 25 & 34 & 50 & 67 \\
\hline$(4,2)$ & & & 1 & 2 & 4 & 7 & 11 & 17 & 25 & 36 & 50 & 70 \\
\hline$(5,1)$ & & & & 1 & 2 & 5 & 8 & 14 & 21 & 32 & 45 & 65 \\
\hline$(6,1)$ & & & & & 2 & 3 & 7 & 11 & 19 & 28 & 43 & 59 \\
\hline$(6,2)$ & & & & & 2 & 3 & 7 & 12 & 19 & 29 & 43 & 61 \\
\hline$(6,3)$ & & & & & 2 & 4 & 8 & $13(\mathbf{1 2})$ & 21 & $31(\mathbf{3 0})$ & 45 & $64(\mathbf{6 2})$ \\
\hline$(7,1)$ & & & & & 1 & 3 & 5 & 10 & 16 & 26 & 38 & 57 \\
\hline$(8,1)$ & & & & & 1 & 2 & 5 & 8 & 15 & 23 & 36 & 52 \\
\hline$(8,2)$ & & & & & 1 & 2 & 5 & 8 & 15 & 23 & 36 & 53 \\
\hline$(8,4)$ & & & & & $1(0)$ & $2(1)$ & $5(4)$ & $9(8)$ & $16(\mathbf{1 5})$ & $25(\mathbf{2 4})$ & $38(37)$ & $56(\mathbf{5 5})$ \\
\hline$(9,1)$ & & & & & 1 & 2 & 4 & 8 & 13 & 22 & 33 & 50 \\
\hline$(9,3)$ & & & & & 1 & 2 & 8 & 4 & 13 & 22 & 33 & 51 \\
\hline$(10,1)$ & & & & & 1 & 2 & 4 & 7 & 13 & 20 & 32 & 47 \\
\hline$(10,2)$ & & & & & 1 & 2 & 4 & 7 & 13 & 20 & 32 & 47 \\
\hline$(10,5)$ & & & & & $1(0)$ & $2(\mathbf{0})$ & $4(\mathbf{0})$ & $7(2)$ & $13(8)$ & $21(\mathbf{1 6})$ & $33(\mathbf{2 8})$ & $49(\mathbf{4 4 )}$ \\
\hline
\end{tabular}

Conjecture 4.10. Let $2 \leqslant N \leqslant 9, t \mid N$, and $(N, t) \neq(6,3),(8,4),(10,5)$. Then the ideal $I_{*}^{N, t}$ is generated by bordism classes of complex $N$-Spin manifolds admitting a semifree circle action of type $t$.

Ochanine [16] proved such a result for Spin manifolds admitting a semifree circle action of odd type whose complex case version corresponds to $(N, t)=(2,1)$ in the above conjecture.

\section{Acknowledgments}

The author is grateful to the anonymous referee for helpful comments which improved the presentation of this paper.

\section{References}

[1] M. N. Ahmad, Cobordism Theory of Semifree Circle Actions on Complex $N$-Spin Manifolds, Ph.D. thesis, Kansas State University, 2011. Available at https://krex.k-state.edu/dspace/handle/2097/12031.

[2] R. Bott and C. Taubes, On the rigidity theorems of Witten, J. Amer. Math. Soc. 2 (1989), 137-186.

[3] P. E. Conner and E. E. Floyd, Differentiable Periodic Maps, Springer Verlag, Berlin, 1964.

[4] P. E. Conner and E. E. Floyd, Maps of odd period, Ann. of Math 84 (1966), $132-156$. 
[5] P. E. Conner and E. E. Floyd, Torsion in SU-Bordism, Mem. Amer. Math. Soc., No. 60, 1966.

[6] A. Dold, Relations between Ordinary and Extraordinary Homology, Colloquium on Algebraic Topology, Aarhus Universitet, 1962.

[7] A. Hattori and H. Taniguchi, Smooth $S^{1}$-actions and bordism, J. Math. Soc. Japan 24 (1972), 701-731.

[8] F. Hirzebruch, Elliptic Genera of Level $N$ for Complex Manifolds, Differential Geometrical Methods in Theoretical Physics, Kluwer, 1988, 37-63.

[9] G. Höhn, Komplexe Elliptische Geschlechter und $S^{1}$ - Äquivariante Kobordismustheorie, Diplomarbeit, Bonn, 1991, arXiv:math.AT/0405232.

[10] R. Jung, Zolotarev-Polynome und die Modulokurve $X_{1}(N)$, Diplomarbeit, Bonn, 1989.

[11] I. M. Krichever, Obstructions to the existence of $S^{1}$-actions. Bordism of ramified coverings, Math. USSR Izv. 10 (1976), No. 4, 783-797.

[12] I. M. Krichever, Generalized elliptic genera and Baker-Akhiezer functions, Math. Notes 47 (1990), 132-142.

[13] P. Landweber, Modular Forms in Algebraic Topology, Lecture Notes in Mathematics, vol. 1326, Springer Verlag, 1988.

[14] K. Liu, On modular invariance and rigidity theorems, J. Diff. Geom. 41 (1995), 343-396.

[15] J. W. Milnor and J. D. Stasheff, Characteristic Classes, Annals of Mathematics Studies, vol. 76, Princeton University Press, 1974.

[16] S. Ochanine, Sur les genres multiplicatifs définis par des intégrales elliptiques, Topology 26 (1987), 143-151.

[17] R. E. Stong, Notes on Cobordism Theory, Princeton University Press, Princeton, 1968.

[18] C. Taubes, $S^{1}$-actions and elliptic genera, Commun. Math. Phys. 122 (1989), $455-526$.

[19] E. Witten, Fermion Quantum Numbers in Kaluza-Klein Theory, Shelter Island II: Proc. 1983 Shelter Island Conf. on Quantum Field Theory and the Fundamental Problems of Physics, MIT Press, Cambridge, Mass., 1985, 227-277.

[20] E. Witten, Elliptic genera and quantum field theory, Commun. Math. Physics 109 (1987), 525-536.

[21] E. Witten, The Index of the Dirac Operator in Loop Space, Lecture Notes in Mathematics, vol. 1326, Springer Verlag, 1988, 161-181.

M. Naeem Ahmad ahmadn@smcsc.edu

Science Division, Spartanburg Methodist College, 1000 Powell Mill Rd., Spartanburg, SC, 29301, USA 